\title{
Robótica y derecho: \\ sugerencias, confluencias, \\ evoluciones en el marco \\ de una investigación europea*
}

\section{ERICA Palmerini ${ }^{* *}$}

Sumario: i. "Robots entre nosotros": de la ciencia ficción a la realidad. II. El robot en la escena jurídica. III. Una infinidad de problemas, ¿un enfoque unitario? A. El antecedente: el proyecto RoboLaw. B. ¿Qué es un robot? Base tecnológica y cuestiones jurídicas. Iv. La identificación de los temas. El robot como objeto (ahora) jurídico. A. De la calificación genérica a la laguna normativa. v. Robot y tutela de los consumidores. vi. Los problemas de la seguridad y de la responsabilidad en el mercado emergente de la robótica. A. Las propuestas de esquemas alternativos a las reglas comunes de la responsabilidad. B. Algunas observaciones críticas. C. Escenarios presentes y futuros. vir. Cyborgs y buman enhancement entre viejo... viII. ... y nuevo.

Este artículo está dedicado al estudioso y querido amigo Édgar Cortés y a su gentil colaboradora, doctora del Ateneo pisano, Indira Díaz Lindao. A ambos les estoy muy agradecida por el interés que mostraron hacia mis investigaciones más recientes acerca de la intersección entre robótica y derecho, por haber albergado este artículo en la prestigiosa revista de la Universidad del Externado y por la infinita paciencia con la que han esperado que estuviera terminado. Traducción del italiano, Indira Díaz Lindao. Título original: "Robotica e diritto: suggestioni, intersezioni, sviluppi a margine di una ricerca europea, publicado" en Responsabilità Civile e Previdenza, fasc. 6, 2016, p. I8I5B. Fecha de recepción: 6 de octubre de 2016 . Fecha de aceptación: 22 de mayo de 2017 . Para citar el artículo: Palmerini, E., "Robótica y derecho: sugerencias, confluencias, evoluciones en el marco de una investigación europea", Revista de Derecho Privado, Universidad Externado de Colombia, n. ${ }^{\circ}$ 32, enero-junio de 2017, 53-97. DOI: https://doi.org/I0.I860I/OI 234366.n32.03

** Doctorado en Derecho. Profesora asociada de la Scuola Superiore Sant'Anna, Pisa, Italia. Contacto: erica.palmerini@sssup.it 


\section{I. "Robots entre nosotros" : de la ciencia ficción a la realidad}

Robots, androides, cyborgs, autómatas, invaden el imaginario contemporáneo y ofrecen ideas para una consideración filosófica y antropológica de las transformaciones que la tecnología imprime al hombre, a su cuerpo y al ambiente que lo rodea. Desde los clásicos de la ciencia ficción, a la filmografía, a las historietas, a los dibujos animados, las sugerencias que ofrecen los distintos géneros literarios, las producciones culturales y los medios de comunicación son tan numerosas que no pueden reproducirse siquiera sumariamente, lo que exime de efectuar citaciones exhaustivas ${ }^{2}$.

Las novedades que nos ofrecen los avances técnicos y científicos de los años (relativamente) más recientes se relacionan con la comprobación (si bien en forma menos espectacular y por algunos aspectos más rudimentaria) de objetos que antes solo eran concebidos por la fantasía de los escritores y por la inspiración creativa de autores cinematográficos y televisivos, diagramadores y diseñadores. Seres artificiales, criaturas híbridas, sistemas cibernéticos sofisticados, que interactúan con el hombre y actúan en el mundo virtual y en el mundo físico; los productos de ingeniería que funcionan con autonomía (al menos en apariencia) ya forman parte de nuestra contemporaneidad, y prácticamente, sin que nos demos cuenta, varios ejemplos de la tecnología robótica han comenzado a entrar en nuestra cotidianeidad.

La automatización está presente desde hace tiempo en el sector industrial, donde cada vez más se hace uso de robots en el ámbito de los procesos productivos, en el ensamble y la manipulación de materiales, en las cadenas de montaje, en la confección de mercancías. No obstante, el sector de la robótica industrial está caracterizado por una rígida separación de las máquinas frente a los trabajadores. El robot industrial está confinado a un espacio estructurado para acogerlo, y aislado por barreras físicas o virtuales. También es conocido el empleo de robots en ambientes extremos e inaccesibles al hombre, como en los planetas del sistema solar o en las profundidades marinas. Rover diseñados por agencias espaciales visitan cuerpos celestes a millones de kilómetros de distancia y pro-

I Robot fra noi. Le creature intelligenti che stiamo per costruire, Turín, 2014 (trad. ital. de Robot Futures, Cambridge, MA, 20I3), es el título de un libro muy divulgado escrito por NourbakHsh, I. R., uno de los más famosos exponentes de la investigación en el campo de la robótica. Otras fuentes recientes de información general sobre el fenómeno son Cingolani, R. y Metta, G., Umani e umanoidi. Vivere con i robot, Bolonia, 2015; De BIAse, L., Homo pluralis. Esseri umani nell'era tecnologica, Turín, 2015.

2 Por otra parte, la reflexión cultural sobre el fenómeno, desde diversas perspectivas disciplinarias, a su vez es tan variada como para hacer prácticamente superfluo, además que arbitrario, cualquier intento que se haga en este sentido en un artículo que afronta el tema desde un aspecto eminentemente jurídico. Se recuerdan tan solo, a título ejemplificativo, Henry, B., Dal Golem ai cyborgs. Trasmigrazioni nell'immaginario, Livorno, 2012; así como los artículos reunidos en el marco del Convenio sobre Roboethics in film, Múnich, 28 y 29 de febrero de 2014, publicados en el volumen homónimo, al cuidado de Battaglia, F. y Weidenfeld, N., Pisa, 20 I4. 
yectan sus imágenes a un centro de control que espera expectante en la tierra; y a todos nos ha pasado seguir por televisión, con partícipe alegría, los inciertos primeros pasos 3 . De otra parte, siniestros drones se emplean en acciones militares, de vigilancia y de intervención bélica, en territorios peligrosos o de difícil acceso. Pero la promesa de la investigación científica en el campo de la robótica es la de introducir a los robots en ambientes corrientes, para desarrollar la función de ayuda doméstica o de asistencia a la persona; transformar el sistema de transporte haciéndolo siempre menos dependiente del control del hombre; ampliar el uso de aviones autónomos o teleoperantes, en el ámbito civil -en la agricultura, en la logística, en el control del territorio, en las redes de carreteras urbanas y ferroviarias, y de las infraestructuras hídricas y energéticas, en la distribución comercial; finalmente, valerse de la tecnología robótica en las actividades de formación y educación.

El sector sanitario y del cuidado de la salud constituye otro escenario de propagación de la innovación robótica ${ }^{4}$, que cuenta entre sus adquisiciones con robots quirúrgicos implementados actualmente en los hospitales, y entre sus objetivos más prometedores, todavía objeto de experimentación, la elaboración de cápsulas médicas inteligentes para llegar a sitios del interior del cuerpo humano; terapia motora, mental, cognitiva y social, asistida robóticamente; sistemas de monitoreo de parámetros fisiológicos robotizados y prótesis biónicas avanzadas implantadas directamente en el sistema nervioso central o periférico. La figura simbólica del cyborg, entidad en parte orgánica, en parte máquina, se traduce en individuos que para superar una discapacidad o una enfermedad, pero con la perspectiva de potenciar sus habilidades físicas e intelectuales, afrontan el implante de componentes mecánico-electrónicos en el cuerpo o en el sistema nervioso.

De las innovaciones que acabamos de mencionar, existen otros y numerosos ejemplos concretos, tanto de productos que se encuentran actualmente en el comercio o de uso corriente, como de productos creados en el laboratorio y sometidos a prueba en ambientes 'protegidos', pero próximos a su puesta en funcionamiento. Aún más vasto y variado es el foco de las innovaciones que, aun sin resultados tangibles, atraen actualmente el interés del jurista, del estudioso de ética aplicada, del economista, del lingüista y del psicólogo. Las perspectivas de las disciplinas comprometidas en la reflexión son múltiples, en razón de la

3 Además de las misiones a Marte de los rover "Sojourner", "Spirity" (http://mars.nasa.gov/mer/ mission/tm-spirit/index.html) y "Opportunity" (http://mars.nasa.gov/mer/mission/status_opportunity.html), todos desarrollados por la NASA, en la misión Rosetta, lanzada por la Agencia Espacial Europea, el lander "Philae" ha "aterrizado" por primera vez sobre un cometa (http:// www.esa.int/Our_Activities/Space_Science/Rosetta/).

4 Una síntesis actualizada de las evoluciones en el sector se encuentra en el informe Healthcare Robotics, 20I4, en Robotics Business Review, Industry Special Report, disponible en: http://www. roboticsbusinessreview.com/research/report/healthcare_robotics_20I4 
complejidad objetiva de la tecnología objeto de estudio y de su predecible impacto, a veces considerado perturbador en el plano socioeconómico. Desde hace relativamente poco la reflexión jurídica pasó a formar parte del debate, pero apareció con un enfoque de lectura que resulta idóneo para cumplir una doble función: por un lado, la de proporcionar un marco de referencia a los operadores del sector, quienes por ciertas razones están preocupados por las implicaciones de su acción y, además, necesitan encontrar un soporte técnico ante potenciales experimentaciones que superen los confines de los laboratorios. Por otro lado, la de inspirar una regulación avanzada que pueda impulsar el surgimiento de la robótica y asegurarle un desarrollo congruente con los valores propios del orden jurídico europeo.

\section{El robot en la escena jurídica}

Hoy, la relevancia económica y social que tiene la tecnología robótica es difícilmente cuestionable. Tanto la prensa como los demás medios de comunicación 5 , así como los numerosos documentos elaborados por instituciones económicas y culturales $^{6}$, subrayan la importancia estratégica de un mercado avanzado de la robótica, predicen sus altos costos comerciales, así como las profundas transformaciones que provocarán en el tejido social, algunas de ellas previstas y deseadas, y otras temidas 7 .

5 The Economist, Special Report, Robots. Immigrants from the future, 29 de marzo, 20 I 4; "Hi, robot. Work and life in the Age of Automation", Foreign Affairs, vol. 94, n. ${ }^{\circ}$ 4, julio-agosto, 20 I 5 . Las técnicas comunicativas empleadas para describir el fenómeno son múltiples: cfr., p. ej., Robotica, una serie de videos con los cuales el New York Times ha ilustrado las transformaciones sociales generadas a partir del surgimiento de los robots (http://www.nytimes.com/video/robotica).

6 Manyika, J., Chui, M., Bughin, J., Dobbs, R., Bisson, P. y Marrs, A. Disruptive technologies: Advances that will transform life, business, and the global economy, McKinsey Global Institute, 20I3, disponible en: http://www.mckinsey.com/insights/business_technology/disruptive_technologies

7 Un ejemplo emblemático de esta ambivalencia se refiere al impacto sobre el mercado. Por un lado, se miran con simpatía las ventajas ofrecidas por la tecnología robótica, bien en el sentido de aliviar a los trabajadores en tareas físicamente gravosas o que pueden comprometer la salud, bien como factor de crecimiento y de creación de puestos de trabajo mediante el desarrollo de un mercado lleno de potencialidades. Por otro lado, se teme que la automatización, siempre creciente, determine una contracción en las formas tradicionales de empleo, principalmente a expensas de los que ocupan puestos de trabajo menos calificados. Un reciente estudio del Pew Research Center, AI, Robotics and the Future of Fobs, agosto de 2014 (http://www.pewinternet. org/2014/o8/o6/future-of-jobs/), reúne de manera emblemática este doble aspecto de la innovación en campo robótico tanto desde la percepción común como de personas expertas. Ver por el contrario, para un desarrollo conceptual del tema, Frey, C. B. y Osborne, M. A., The future of employment: How susceptible are job to computerization?, I 7 de septiembre, 2013, disponible en: www.oxfordmartin.ox.ac.uk; además, para un análisis documentado de teoría económica, Brynjolfsson, E. y McAfee, A., Race against the Machine: How the Digital Revolution is Accelerating Innovation, Driving Productivity, and Irreversibly Transforming Employment and the Economy, Lexington, $20 \mathrm{I}$; M. Ford, Rise of the Robots: Technology and the Threat of a Fobless Future, New York, 2015 . 
Paralelamente a la difusión de las aplicaciones robóticas y a su empleo en diversos sectores, pero sobre todo con miras a su introducción en la vida cotidiana, emergen preocupaciones de orden ético y jurídico. Desde el punto de vista ético son cuestionados, principalmente, los aspectos relativos a la aceptabilidad social de la innovación tecnológica, el acceso a la misma según criterios no discriminatorios y fundados en principios de justicia social, y la integridad de la investigación científica en terrenos límite. Pero como estos aspectos no son propios de la reflexión ética y filosófica de la robótica, sino del debate crítico sobre la innovación tecnológica en general, la discusión asume diversos puntos de vista y se concentra sobre temas característicos de este sector. La roboética ${ }^{8}$ es principalmente la ética de los investigadores, de los fabricantes y de las personas involucradas en el uso de los robots ${ }^{9}$. Una de sus ramas específicas estudia la ética de la conducta humana en la interacción con artefactos robóticos y el impacto que este vínculo genera en los planos de la identidad y de lo emocional ${ }^{\text {Io }}$; sobre la base de que los robots son máquinas diseñadas frecuentemente para evocar, a partir de similitudes y de reacciones a los estímulos del ambiente, a un ser viviente, humano o animal ${ }^{\mathrm{I}}$, con las consecuencias que generan, por un lado, la ficción y la simulación de emociones ${ }^{\mathrm{I} 2}$, y por el otro, la proyección de significados en las relaciones ${ }^{13}$.

8 El término ha sido acuñado por Veruggio, G., The EURON Roboethics Roadmap, 2006, disponible en: http://www3.nd.edu/ rbarger/ethics-roadmap.pdf. Ver también VerrugGio, G. y Operto, F., "Roboethics: Social and Ethical Implications of Robotics", en Handbook of Robotics, Siciliano, B. у Кнатів, О. (eds.), 2008, Springer, i I 5 I ss.

9 Lin, P., Abney, K. y Bekey, G. A. (eds.), Robot Ethics. The Ethical and Social Implications of Robotics, Cambridge (MA), 20 2; Id., "Robot Ethics: Mapping the issues for a mechanized world", en Artificial Intelligence, 20 i I, I 75 (5-6), 942-949; Capurro, R. y Nagenborg, M. (eds.), Ethics and Robotics, Heidelberg, 2009; Decker, M. y Gutmann, M. (eds.), Robo- and Information Ethics. Some fundamentals, Berlin, 20I 2; Wallach, W., "From Robots to Techno Sapiens: Ethics, Law and Public Policy in the Developments of Robotics and Neurotechnologies", en Law, Innovation and Technology, 20I I, 3(2), I85-207; von Schomberg, R., "From an ethics of technology towards an ethics of knowledge policy: Implications for robotics", en Artificial Intelligence and Society, 2008, 22, 33 I-348. Con respecto específicamente a la neurobótica, WAGNER, J. J. CANNON, D. M. y van Der Loos, H. F. M., Cross-cultural Considerations in Establishing Roboethics for Neuro-robot Applications, Proceedings of the 2005 IEEE $9^{\text {th }}$ International Conference on Rehabilitation Robotics, Chicago, IL, usa; R. Bogue, "Robot Ethics and Law", en Industrial Robot: An International fournal, 2014, 4I(5), 398-402.

io Turkle, S., Alone Together. Why We Expect More from Technology and Less from Each Other, New York, $20 \mathrm{I}$ I.

I I Es el caso, p. ej., del robot "Paro" (http://www.parorobots.com/), que tiene la apariencia de una cría de foca y es usado para la terapia de personas ancianas y con problemas cognitivos porque ayuda a reducir el estrés y facilita la socialización. Ver, p. ej., "Japan develops robotic seals to comfort sick and elderly", ввс, 2 de octubre, 2010 (http://www.bbc.com/news/ health-I I459745).

I 2 Sharkey, A., "Robots and human dignity: A consideration of the effects of robot care on the dignity of older people", en Ethics and Information Technology, 20 I4, I6, 63-75.

I 3 Ver, p. ej., Sharkey, N. y Sharkey, A., "The crying shame of robot nannies: An ethical appraisal", en Interaction Studies, 20 io, i I (2), I6 I-i 90; Sharkey, A. y Sharkey, N., "Granny and the 
Por otra parte, la ética de las máquinas ${ }^{\mathrm{I}}{ }^{4}$ se preocupa por construir e insertar en el funcionamiento de las máquinas un código moral. Si los robots son eficientes en cuanto (también) caracterizados por una autonomía que se alimenta por medio de la capacidad de aprendizaje de la que son dotados y los habilita al cumplimiento de acciones no programadas, resulta importante incluir en el código del programa reglas que puedan gobernar los comportamientos de los robots, aun los no anticipados, permitiéndoles distinguir, entre las conductas posibles, la más apropiada o menos nociva. Esta rama de estudio presenta, de un lado, un perfil esencialmente tecnológico, que debe proporcionar las soluciones técnicas que estén en capacidad de dotar al robot de una moral, y del otro, un perfil de reflexión ética, que se ocupa de construir un conjunto ordenado y simple de reglas de comportamiento, según la sugestión de las leyes de Asimov, que posteriormente puedan ser transformadas en un código binario.

Por su parte, el interés del jurista se suscita por la entrada de los robots en ambientes cotidianos y por la variedad de relaciones que de ellos se derivan y que, como es posible imaginar, serán la regla en el futuro. Las modalidades de interacción son distintas entre sí -daños, relación contractual, injerto o implante en el cuerpo- y evocan otras ramas del sistema jurídico. El robot diseñado para la asistencia en el hogar o en una clínica hospitalaria, entra en íntimo contacto con la persona que lo utiliza (con frecuencia un paciente o, en general, una persona en condiciones de fragilidad debido a su edad, a una enfermedad o a una incapacidad), con sus familiares y con los operadores de ese ambiente, suscitando de forma inevitable la ocasión para la ocurrencia de accidentes, lesiones o perjuicios. Una elevada capacidad sensorial del robot y todas las especificaciones técnicas incluidas en el diseño de la máquina pueden minimizar, aunque no anulen completamente, la eventualidad de colisiones, caídas e impactos de diversa índole. Además, cuanto más delicada es la función que deben llevar a cabo (como, p. ej., levantar a un paciente de la cama), tanto más graves pueden ser las consecuencias dañosas provocadas por un mal funcionamiento, por una errónea interpretación de los estímulos ambientales o por un desperfecto eléctrico. Así, el robot quirúrgico que permite efectuar intervenciones de alta precisión y operar en dimensiones muy pequeñas, sin la dificultad que eventualmente tendría el

robots: Ethical issues in robot care for the elderly", en Ethics and Information Technology, 20 I 2, I 4 (I), 27-40; Scheutz, M., "The Inherent Dangers of Unidirectional Emotional Bonds between Humans and Social Robots", en Robot Ethics: The Ethical and Social Implications of Robotics, cit., 205 SS.

I4 Anderson, M. y Anderson, S. L., Machine Ethics, Cambridge, 20 i i; Gunkel, D. J., The Machine Question. Critical Perspectives on AI, Robots, and Ethics, Cambridge (Mass.), 20 I ; Wallach, W. y Allen, C. Moral machines: Teaching robots right from wrong, Oxford-New York, 2009; Malle, B. F., Scheutz, M. y Austerweil, J. L., Networks of Social and Moral Norms in Human and Robot Agents, International Conference on Robot Ethics, Lisbon, 20 I 5 ; Malle, B. F. y Scheutz, M. Moral Competence in Social Robots, IEeE International Symposium on Ethics in Engineering, Science, and Technology, Chicago (IL), 2014. 
cirujano en el plano visual y manual, puede causar lesiones a los propios órganos y a los vasos sanguíneos circundantes, si por error el software operativo genera movimientos involuntarios.

Pero la modalidad de la interacción dañosa es solo una de las tantas posibilidades. Software robots (softbots) son comúnmente usados para realizar transacciones financieras ${ }^{15}$; sistemas automatizados llevan a cabo ventas on line y permiten organizar la distribución de los bienes adquiridos; sistemas expertos (o deep learning systems) ${ }^{\mathrm{I} 6}$ constituyen un auxilio potente, en contextos clínicos, en el diagnóstico de una condición patológica a partir de la observación de las condiciones del paciente ${ }^{17}$; softwares son utilizados para escribir artículos periodísticos en circunstancias de emergencia, como por ejemplo, para reportar un sismo en tiempo real ${ }^{18}$. La legalidad de este tipo de aplicaciones tecnológicas y de las operaciones realizadas a través de ellas surge del tipo de relación contractual establecida o mediada por un ente robotizado; del instrumento y del proceso decisorio que conduce a la elección terapéutica, o de la dimensión del posible reconocimiento por atribuir al trabajo (que llamaremos) intelectual, generado por un robot. Otra realidad próxima, con la que el derecho también deberá confrontarse, es la de las transacciones corrientes, incluso de modesto valor, gracias a las cuales un robot puede comprar productos alimenticios o medicinas para la persona a la que cuida ${ }^{19}$.

En definitiva, la prospectiva de la compenetración entre el hombre y la máquina se lleva a cabo mediante una serie de aplicaciones, que van desde el implante de prótesis avanzadas, a la creación de conexiones cerebro-máquina para controlar el movimiento de una extremidad o de un robot (o incluso para exteriorizar el pensamiento), a la estimulación cerebral profunda. Estas transformaciones inducen a revisar la construcción jurídica del concepto de cuerpo y de sus partes, y a determinar, sobre bases nuevas, las fronteras operativas de la noción de integridad física, así como a replantear el régimen aplicable a realidades materiales, ya sean externas o internas, que desarrollan funciones esenciales para la persona.

I 5 Acerca de los riesgos vinculados al creciente uso de sistemas de inteligencia artificial y de programas automáticos en las finanzas modernas, transformadas de esta manera en una "cyborg finance" en la que los principales actores son en parte no humanos, ver Lin, T. C. W., The New Investor, 6o UCLA L. Rev. 678 (2012-2013).

I6 Un ejemplo es Watson de la Івм: http://www-03.ibm.com/innovation/us/watson/

i 7 Ferrucci, D., Levas, Al., Bagchi, S., Gondek, D. y Mueller, E. T. , "Watson: Beyond Jeopardy!", en Artificial Intelligence, 201 3, 93-105.

I 8 Ver: http://www.bbc.com/news/technology-266I405 I

I9 El proyecto europeo Robot-ERA (http://www.robot-era.eu/robotera/) prevé el desarrollo de tres robots personales: el primero es un robot de asistencia doméstica, el segundo se mueve dentro de un inmueble compuesto por varias unidades habitables, por ejemplo, un condominio, y el tercero es utilizado para la circulación en el espacio externo, donde puede cumplir tareas simples. 


\section{Una infinidad de problemas, ¿̨un enfoque unitario?}

Las posibles interacciones entre derecho y robótica han evidenciado, junto con la multiplicidad de las aplicaciones tecnológicas mencionadas, la variedad de problemas jurídicos que estas generan y la dificultad de reconducirlas hacia un paradigma homogéneo.

Al tiempo que crece el interés de las ciencias sociales por este sector en expansión, también surge la duda sobre la posibilidad de que la robótica se constituya en objeto de estudio autónomo por parte del jurista. En concreto, parece plantearse la objeción a un tratamiento del tema en términos de "excepcionalidad", como ha sucedido en otros ámbitos ${ }^{20}$, es decir, la pretensión de considerar los problemas generados por el impacto de la robótica como inéditos y cualitativamente diversos de los planteados por otras categorías, o bien susceptibles de ser considerados como un complejo homogéneo de problemáticas, a ser reglamentadas, eventualmente, por medio de un cuerpo unitario de reglas ${ }^{21}$.

La crítica refleja la experiencia que acompañó el nacimiento del derecho cibernético, del derecho de internet, definido en su inicio tan útil como un "derecho de los caballos" ${ }^{2}$. Sigue siendo válida la enseñanza según la cual todo fenómeno nuevo, especialmente cuando está ligado al desarrollo científico y tecnológico, no requiere necesariamente de la introducción de normas nuevas, ante

Por ejemplo, con la expresión "genetic exceptionalism" se ha indicado, generalmente en tono crítico, la actitud de tratar la información y los datos genéticos como si estuvieran dotados de características especiales frente a los meros datos personales y a los datos sanitarios en general y, por lo tanto, fueran merecedores de una disciplina distinta y de una protección más intensa. Ver, al respecto, Suter, S. M., "The Allure and Peril of Genetic Exceptionalism: Do We Need Special Genetics Legislation?", 79 Wash. Univ. L. Quart. 669 (2001); Murray, T. H., "Genetic Exceptionalism and 'Future Diaries': Is Genetic Information Different from Other Medical Information?", en Genetic Secrets. Protecting Privacy and Confidentiality in the Genetic Era, Roтнstein, M. A. (ed.), New Haven, I997, 64 ss.; RothsteIn, M.A., Why Treating Genetic Information Separately is a Bad Idea, 4 Texas Rev. L. \& Politics 33 (1999); Gostin, L. O. y Hodge, J. G., Genetic Privacy and the Law: An End to Genetics Exceptionalism, 40 Furimetrics 7. 3 I (1999); Wertz, D. C., Society and the Not-So-New Genetics: What Are We Afraid of? Some Future Predictions from a Social Scientist, I3 7. Contemp. Health L. \& Policy 3 I3 (1997); Holm, S., There Is Nothing Special about Genetic Information, en Genetic Information. Acquisition, Access, and Control, Thompson, A. K. y Chadwick, R. F. (eds.), New York, I999, 97 ss.

2 I Acerca del robotic exceptionalism, del impacto sistemático que la robótica podría tener sobre el derecho, y de la utilidad, contraria a las opiniones escépticas, de desarrollar una reflexión integral sobre robótica y derecho, se expresa CaLo, R. M., Robotics and the Lessons of Cyberlaw, California Law Review, 2015, I03, 5 I3 ss., spec. 553 ss.

22 Ver las posturas de EаsтеRввоок, F. H., Cyberspace and the Law of the Horse, University of Chicago Legal Forum, I996, 2017, y Lessig, L. The Law of the Horse: What CyberLaw Might Teach, Harvard Law Review, I999, I I3, 50I. El primero observó, en la apertura de un convenio sobre la cyberlaw, que de esta se tiene necesidad como se tiene necesidad de un "derecho de los caballos": estos son comúnmente objeto de contratos de compraventa, pueden dar lugar a obligaciones resarcitorias si al patear hieren a un transeúnte, el veterinario que los atiende debe actuar con diligencia. Sin embargo, la legalidad que les compete no debe originar una nueva y autónoma rama del derecho. 
la posibilidad de ser regulado por el sistema existente, capaz de adquirir una gran elasticidad.

Ciertamente, cuando se desarrollan los discursos sobre derecho y robótica no se pretende proponer la creación de un cuerpo completo de regulaciones sobre bases mayoritariamente renovadas, ni instituir el nacimiento de una nueva rama del saber jurídico. Se trata, sobre todo, de evaluar si los avances que se están llevando a cabo en la investigación robótica y en la puesta en funcionamiento de sistemas innovativos dan lugar a una eventual fricción con las reglas existentes, minando su justificación racional o su efectividad.

Un estudio orgánico de los núcleos temáticos que encierra el binomio derecho y robótica representa, entonces, una articulación lineal de la reflexión jurídica sobre el vínculo entre el derecho y las tecnologías emergentes, que se va desarrollando y sedimentando cada vez en mayor medida, sin pretensiones de exhaustividad ni ambiciones sistemáticas.

Entre las circunstancias que justifican un tratamiento unitario del tema, al menos prima facie, se encuentra, principalmente, una cuestión fáctica, esto es, el hecho de que las múltiples aplicaciones estudiadas pertenecen a un mismo sector de la investigación y de la innovación tecnológica -la robótica- que, aunque se vale de diversas disciplinas para poner en funcionamiento sus productos, presenta características suficientemente específicas y propias. En segundo lugar, la relevancia es de carácter socioeconómico, puesto que la robótica implica un mercado en expansión, considerado estratégico sobre el plano económico y de gran impacto a nivel social, cuyo crecimiento requiere ser controlado mediante una intervención regulatoria. De estos aspectos emerge la razón principal que alimenta este estudio. Un marco de reglas claras que pueda conferir certeza respecto de los deberes y de las responsabilidades de los actores involucrados en el proceso de innovación ${ }^{23}$; en este sentido, la demanda de regulación proviene de los mismos operadores del sector, quienes requieren adaptar sus estrategias, y planos de investigación y de inversión a las mismas.

De manera adicional, el estudio también puede servir para dirigir el sentido del desarrollo del mercado y garantizar que ello ocurra en un contexto de respeto de los derechos fundamentales. La creciente sensibilidad por las implicaciones éticas y jurídicas de la innovación tecnológica, según una actitud eficazmente

23 Esta percepción es compartida en numerosos documentos que analizan el desarrollo potencial del sector y del mercado correspondiente, e identifican la ausencia de regulación como una de las barreras, de tipo no tecnológico, respecto al advenimiento de un mercado avanzado de la robótica: ver, p. ej., sPARc, Multi-annual Roadmap for Robotics in Europe, 6.2.20I5, 77; UK Robotics and Autonomous Systems Special Interest Group (RAS-SIG), RAS 2020. Robotic and autonomous systems, julio, 20 I4, 7, disponible en: https://connect.innovateuk.org/documents/29030 I 2/I6074728/RAS\% 20UK\% 20Strategy?version=1.0 
condensada en la fórmula de responsible research and innovation (RRI) ${ }^{24}$, se encuentra cada vez más respaldada por la conciencia de que un ambiente legal claro, transparente y predispuesto a recoger las novedades apenas delineadas, puede constituir un incentivo al desarrollo de este sector del mercado, contrario a una falaz pero enérgica representación de la regulación jurídica en términos de impedimento o freno a la innovación.

En definitiva, este enfoque unitario y proactivo se encuentra reafirmado por la reciente posición adoptada por el regulador europeo. Un grupo de trabajo instituido por la Comisión de Asuntos Jurídicos del Parlamento Europeo redactó un documento analítico sobre los problemas jurídicos planteados a partir de las evoluciones en el campo de la robótica, que, con un llamado para la expedición de una resolución del mismo Parlamento, invita a la Comisión a adoptar una regulación en la materia ${ }^{25}$. Como tema de interés académico, la regulación de la robótica se ha incluido en la agenda de elaboración de políticas.

\section{A. El antecedente: el proyecto RoboLaw}

Entre las motivaciones de esta investigación se encuentra una segunda circunstancia, más ocasional, esto es, la individualización de los problemas en el contexto de una investigación desarrollada en el ámbito del Séptimo Programa Marco y llevada a cabo por un grupo interdisciplinario ${ }^{26}$. Esta se inscribe en una colaboración entre juristas e ingenieros; inicialmente estuvo focalizada en el análisis de casos de estudio individuales (p. ej., los problemas jurídicos planteados por la experimentación de un robot para la recolección de residuos urbanos, al que se le había permitido circular por las calles de Peccioli, un pequeño pueblo de la provincia de $\mathrm{Pisa}^{27}$ ) y, sucesivamente, adoptó una postura abierta a la considera-

24 El concepto de RRI es, en realidad, mucho más complejo y no se agota en la preocupación por la ética de la investigación y de sus aplicaciones. Para una visión general en el contexto de las políticas europeas que sirven de sostén a la ciencia y a la tecnología, ver European Commission, Options for Strengthening Responsible Research and Innovation, Luxembourg, 20 I 3. Para una valoración del nivel de penetración de los cánones de la RRI en la investigación científica en el campo de la robótica, ver Koops, B.-J., Palmerini, E. y SAlvini, P. Responsible Innovation and Robotics, en Handbook - Responsible Innovation: A Global Resource, R. von Schomberg (ed.), en proceso publicación por Edward Elgar Publishing.

25 Committee on Legal Affairs, Draft Report with recommendations to the Commission on Civil Law Rules on Robotics (20I5/2 IO3 (INL), 24 de enero de 20I 7. El Parlamento aprobó la propuesta de Resolución el in de febrero de 2017.

26 RoboLaw - Regulating Emerging Robotic Technologies: Robotics Facing Law and Ethics (G.A. n. 289092), 201 2-2014, disponible en: www.robolaw.eu

27 Se alude al proyecto europeo Dustbot - Networked and Cooperating Robots for Urban Hygiene (www.dustbot.org), 2006-2009. Sobre las implicaciones jurídicas, ver SALvini, P., et al., $A n$ Investigation on Legal Regulations for Robot Deployment in Urban Areas: A Focus on Italian Law, en Advanced Robotics, 2010, 24, I90I-I9I7. 
ción de todas las potencialidades, aunque también de los riesgos, generados por el progreso de la investigación y por los avances de la industria robótica.

El proyecto fue estructurado sobre cuatro líneas de análisis: la primera estuvo dirigida a estudiar el rol de las distintas fuentes de la regulación de la ciencia y la tecnología. En el contexto del tema más amplio de la governance de las tecnologías emergentes, la investigación se focalizó sobre las ventajas y los límites de los instrumentos de soft law superpuestos a la legislación vinculante; además, en un campo dominado por una normatividad de origen técnico se afrontó el problema clave del avance de la regulación privada ${ }^{28}$. Las reglas técnicas y estándar emanadas por organismos privados, algunas veces, aunque no siempre, en razón de una remisión legislativa explícita, fueron examinadas en sus aspectos críticos, ligados tanto a la legitimación democrática de los entes que imparten las prescripciones como a la accesibilidad y a la imparcialidad de sus contenidos; principalmente, se analizó su complejo vínculo con los deberes de seguridad y de conformidad para el uso, que pesa sobre los fabricantes y distribuidores, y sus repercusiones en el plano de la responsabilidad ante la observancia o inobservancia de tales preceptos ${ }^{29}$.

La definición de la base tecnológica de la investigación, a través de la construcción de una taxonomía de las aplicaciones robóticas (estructurada sobre los elementos de la materialidad, del nivel de autonomía, de la función, del ambiente y de la interacción del hombre) y la descripción del estado del arte en materia, constituyó un segundo tramo de la investigación ${ }^{3 \circ}$.

Un tercer segmento fue dedicado a la descripción del debate ético-jurídico sobre el tema del buman enbancement, en primer lugar, reconstruido en sus aspectos generales, y luego discutido en relación específica con su potencialización por medio de las técnicas robóticas ${ }^{3 \mathrm{I}}$.

La investigación propiamente jurídica, inicialmente, giró en torno a la delineación de las zonas de intersección e influencia recíproca entre derecho y ro-

28 Esta parte de la investigación confluyó en el volumen Law and Technology. The challenge of regulating technological development, Palmerini, E. y Stradella,E. (eds.), Pisa, 2013, que reúne las Actas del Convenio "Regulating technological development at the intersection of science and law", Pisa, 2 I y 22 de junio de 2012 . Sobre el vínculo complejo entre innovación y regulación en el sector de la robótica ver, en particular, PaLmerini, E., "The interplay between law and technology, or the RoboLaw project in context", ibíd., 7-24.

29 Ver, en particular, en el volumen citado en la nota precedente, el análisis de ZEI, Astrid, "Shifting the boundaries or breaking the branches? On some problems arising with the regulation of technology", I67-204.

30 Salvini, P., Deliverable D4.I - Taxonomy of robotic technologies; Salvini, P. y ShaH, H., Deliverable D4.2 - State-of-the-art in robotic research: Case-studies from SSSA laboratories.

3 I Son referentes en este aspecto el informe interno de Battaglia, F. y Carnevale,A., Deliverable $\mathrm{D}_{5 . \mathrm{I}}$ - An ethical-philosophical analysis of the human enhancement; y los volúmenes Beyond Therapy v. Enhancement? Multidisciplinary analyses of a heated debate, Lucivero, F. y VedDer, A. (eds.), Pisa, 2013; Reframing the Debate on Human Enhancement, Battaglia, F. y Carnevale, A. (eds.), HumanaMente, n. ${ }^{\circ}$ especial 26, 2014. 
bótica $^{32}$, para luego focalizarse, por un lado, sobre la discusión interdisciplinaria de los problemas considerados de mayor relevancia, como el de la responsabilidad33; y sobre el análisis de aplicaciones robóticas singulares, identificadas por su inminencia, por la novedad de las cuestiones que plantean y por la importancia de su impacto social ${ }^{34}$. El resultado de esta selección llevó a centrar la atención en los robots quirúrgicos, las prótesis robóticas, los vehículos autónomos y los care robots, que han sido examinados considerando todas sus implicaciones éticas y jurídicas. Los resultados de la investigación han constituido la base para la formulación de recomendaciones de políticas recogidas en un documento final destinado a la Comisión Europea35.

El enfoque analítico adoptado por la redacción de este documento refleja la extrema diversidad de las aplicaciones robóticas, circunstancia que hace imposible una representación unitaria, y tampoco justifica tentativos de tratamientos omnicomprensivos, especialmente cuando se orientan al objetivo pragmático de proponer soluciones de regulación que correspondan a efectivas lagunas del sistema o puedan remediar ineficiencias o encuadramientos discutibles.

\section{B. ¿Qué es un robot? Base tecnológica y cuestiones jurídicas}

La dificultad de individualizar una matriz unitaria del discurso también reside en la ausencia de una definición unívoca de robot en el plano tecnológico. La imposibilidad sustancial de compartir una noción suficientemente precisa, que responda a las múltiples formas de implementación robótica existentes, es una constatación común y un punto de partida constante, aunque negativo, de cualquier reflexión sobre el tema ${ }^{36}$. En la búsqueda de un rasgo característico, el acento se pone con frecuencia en la capacidad de los robots de ejecutar tareas de manera automatizada, o bien en la autonomía de la máquina frente al control humano, en la movilidad en el ambiente o, incluso, en el dato exterior de las

32 LeEnes, R. et al., Deliverable D $3 . \mathrm{I}$ - Inventory of current state of robolaw.

33 Rethinking Responsibility in Science and Technology,Battaglia, F., Mukerji, N. y Nida-Rümelin, J. (eds.), Pisa, 2014.

34 El método de selección de cuatro aplicaciones robóticas tiene en cuenta el proceso de clasificación formulado y aplicado en el ámbito del proyecto europeo EthicBots: Tamburrini, G. y Datteri, E. (eds.), Deliverable D 2 - Methodology for the identification and analysis of technoethical issues (http://ethicbots.na.infn.it/).

35 Palmerini, E. et al., Deliverable D6.2 - Guidelines on regulating robotics. Una síntesis de los resultados del proyecto se encuentra también contenida en Bertolini, A. y Palmerini, E. Regulating Robotics: A Challenge for Europe, en Upcoming issues of EU Law. Compilation of in-depth analyses. Workshop for the IURI Committee of the European Parliament, Session II, 94-I 29, Brussels, Office for the Official Publications of the European Communities, 20r4, disponible en: http:// www.europarl.europa.eu/studies

$36 \mathrm{Al}$ igual que la mención al origen del término robot, que habría sido usado por primera vez por el dramaturgo ciego Karel Căpek en su trabajo Rossum's Universal Robots, como derivación de la raíz robota que significa "trabajo duro, trabajo forzado". 
figuras antropomorfas. A pesar de su generalidad y su focalización en un aspecto considerado central, ninguna de estas propuestas puede acoger los numerosos y diversos aspectos de los productos robóticos. Por ejemplo, un robot quirúrgico tiene la apariencia de un aparato sofisticado, pero no es autónomo, sino que es manejado por el cirujano aunque sea a través de una interfaz "inteligente"; cuenta con brazos móviles, pero sin embargo está adherido a la plataforma operatoria; un vehículo autónomo tiene el aspecto de un automóvil normal, aunque se encuentre en capacidad de moverse sin el control de una guía; mientras que robots usados con fines educativos o de entretenimiento pueden tener el aspecto de animales o exteriorizar su naturaleza sin evocar la forma humana.

No obstante esta ambigüedad, existe un acuerdo sustancial en identificar las características distintivas de los robots, desde un punto de vista técnico, en la capacidad de recoger datos mediante sensores (sense), de procesar los datos en bruto (think), de planificar y cumplir acciones mediante conocimientos e informaciones adquiridas, generalmente, en función de objetivos prefijados (act). Por su parte, características solo eventuales son la capacidad de comunicación con un operador, con otros robots o con una red externa, y la de aprendizaje ${ }^{37}$. Realmente es posible observar una línea de continuidad entre la máquina automática y el robot propiamente dicho, que se diferencia de la primera, sustancialmente, por una complejidad mucho mayor, más que por una delimitación neta de orden cualitativo ${ }^{38}$. Avances en el campo de la percepción, de la representación del espacio, del reconocimiento de objetos, rostros y situaciones, de la planificación del movimiento y de la integración de estas funciones convierten a los robots actuales en instrumentos siempre más eficaces para el desarrollo de diversas tareas, en contextos no predeterminados.

Esta definición esencial reenvía a las características intrínsecas de la tecnología robótica que también son interesantes desde el punto de vista jurídico. El robot, en cuanto entidad dotada de un aspecto físico, aunque también de un sistema de software que procesa información, presenta la potencialidad y los riesgos de ambos mundos, el físico y el digital. Combinando estos dos rasgos característicos, en particular, se está en capacidad de asegurar el desarrollo de un amplio espectro de funciones útiles, pero también de exponer al usuario, así como a otras personas, al riesgo de lesiones en caso de interacción defectuosa en la esfera física y moral. La posibilidad de afectar los intereses de las personas, o de causarles pérdidas económicas, constituye una de las principales razones por las que la difusión de los robots adquiere relevancia jurídica. La conectividad es otra

37 Commission de réflexion sur l'Éthique de la Recherche en sciences et technologies du Numerique d'Allistene (CERNA), Éthique de la recherche en robotique, 2014 , I 2.

$3^{8}$ Por ejemplo, una heladera puede activar el motor cuando percibe una temperatura superior a la establecida, pero funciona sobre la base de la percepción de un único dato (temperatura) que desencadena una única acción: encender o apagar un interruptor. 
característica frecuentemente presente en los sistemas robóticos, en virtud de la cual el robot se comunica mediante la red de internet con otros robots, o intercambia datos en ambos sentidos con el fabricante o el distribuidor. Esta característica depende de la necesidad de ampliar los conocimientos a disposición del robot particular, sin sobrecargar su sistema operativo. Los robots no poseerán, al menos en una fase inicial, habilidades sofisticadas de percepción e interpretación del ambiente que los rodea; para ser capaces de reconocer objetos, rostros, y de ejecutar tareas no predeterminadas con antelación, deberán recurrir a los recursos online y luego, eventualmente, compartirlos con otros robots. Naturalmente, este elemento distintivo, sumado al hecho de que determinados tipos de robots, como el vehículo autónomo o el robot asistente, operan en un contexto privado y están en contacto continuo con la persona que los utiliza, genera inmediatas preocupaciones ligadas a la tutela de la privacy; al mismo tiempo que constituye un posible factor de expansión de la responsabilidad en la medida en que permite evidenciar situaciones de riesgo.

La capacidad de actuar con autonomía o, incluso, de aprender de la experiencia o de actualizar el propio sistema operativo de manera no controlada, se encuentra, por otra parte, en los albores de una posible fricción con los criterios comunes de imputación de la responsabilidad por los daños causados por cosas o productos y, por lo tanto, merecen ser analizados a fondo en relación con su impacto real sobre el actual sistema.

\section{La identificación de los temas. El robot como objeto (ahora) jurídico}

La variedad de las aplicaciones tecnológicas que el sector de la robótica está desarrollando, y la dificultad misma de ofrecer una definición unívoca de robot, que no sea ni demasiado inclusiva ni muy circunscrita, evidencian un tentativo de seleccionar algunos temas que contribuyen a resaltar la relevancia del objeto de estudio en el discurso jurídico.

La idea según la cual, a la calificación de los robots como máquinas, y en forma más genérica como productos destinados al consumo, debe seguir la aplicación de una serie de regulaciones (preponderantemente de matriz europea) dirigidas, principalmente, a asegurar estándares mínimos de seguridad para su puesta en el comercio, es totalmente preliminar 39 . La Directiva relativa a las máquinas 2006/42/CE, así como la Directiva 2001/95/CE sobre la seguridad general de los productos, establecen los requisitos de seguridad que un fabricante debe respetar para la puesta en el comercio de sus productos, previendo

39 Acerca de esta perspectiva de análisis, ver Santouosso, A., Boscarato, C. y Caroleo, F., Robot e diritto: una prima ricognizione, en Nuova giur. civ. comm., 20 I 2, II, 494 ss.; aunque también LEENEs, R., Deliverable D $3 . \mathrm{I}$ - Inventory of current state of robolaw, cit., 35 ss. 
el tipo de información a proporcionar a los consumidores (p. ej., mediante el folleto de instrucciones) y regulan la colocación de la marca CE. La definición de las medidas de seguridad está a cargo de un sistema complejo que compete, en parte, a las autoridades nacionales que fijan los procedimientos y las condiciones con base en las cuales un producto puede ser considerado seguro; y en parte, a la determinación de los organismos internacionales de seguridad, mediante la fijación de los estándares técnicos ${ }^{4}$.

El hecho de que el producto pertenezca, eventualmente, a una clase más específica reenvía a otras regulaciones. Es el caso de los robots que constituyen dispositivos médicos como las prótesis, las nanocápsulas robóticas, los exoesqueletos utilizados en el ámbito sanitario y los robots quirúrgicos. En este caso, la normatividad aplicable es proporcionada por las dos directivas sobre la materia ${ }^{4 \mathrm{I}}$ y por las respectivas normas de implementación que trazan un cuadro completo de regulación, que en todo caso se presenta en un nivel extremadamente general e insuficiente para captar los riesgos y las potencialidades de estos productos ${ }^{42}$. Dicha normatividad es considerada como un marco regulatorio favorable a la innovación en el ámbito sanitario, toda vez que la uniformidad de reglas respecto de cualquier dispositivo de uso médico permite que los fabricantes se puedan mover en diversos sectores del respectivo mercado, sin incurrir en costos transaccionales elevados.

Por otra parte, la naturaleza transversal y general de la disciplina le impone situarse en un nivel mínimo de seguridad, al cual podrían escapar justamente los dispositivos tecnológicamente más avanzados. De hecho, las principales debilidades de este esquema regulatorio tienen que ver, por un lado, con la falta de consideración de los nuevos riesgos que plantea la creciente sofisticación de los dispositivos ${ }^{43}$; y por el otro, con la insuficiente regulación de la fase experimental y de puesta en funcionamiento de los dispositivos, los cuales son tomados en consideración, casi exclusivamente, al momento de su introducción en el mercado. Respecto al primer aspecto, no se consideran las características especiales de los dispositivos robóticos con un nivel elevado de integración en el cuerpo, por

40 Véanse, por ejemplo, los estándares Iso IO2 I 8-I/2, Robots and robotic devices. Safety requirements for industrial robots (Part I: Robots; Part 2: Robot Systems and Integration), Ginebra, 201 I; e ISO I3482, Robots and robotic devices - Safety requirements for service robots - Personal care robot, Ginebra, 2014.

4I Directiva 93/42/CEE del Consejo, del I4 de junio de I993, acerca de los dispositivos médicos; Directiva 90/385/CeE del Consejo, del 20 de junio de I990, sobre los productos sanitarios implantables activos.

42 Para un análisis completo del tema, ver Palmerini, E., A legal perspective on body implants for therapy and enhancement, en International Review of Law, Computers \& Technology, 201 5, 29 (2-3), 226-244.

43 Se trata de un fenómeno característico de "regulatory disconnection", según la nomenclatura empleada por Brownsword, R. y Goodwin, M., Law and the Technologies of the Twenty-First Century, Cambridge, 201 2, 369 ss. 
ejemplo, mediante la conexión al sistema nervioso, central o periférico, y la posibilidad de una comunicación Wireless entre el dispositivo y un operador u otra plataforma externa. La Directiva 90/385 se ocupa principalmente de los dispositivos implantables, como los pacemakers o los infusores para la insulina, pero la época en que se expidió la hace obsoleta, tanto en relación con las potencialidades invasivas de los nuevos dispositivos como con la extrema delicadeza y complejidad de las funciones con las que están destinadas a interferir. La disciplina es igualmente inadecuada respecto de los riesgos de interferencia externa derivados de la conectividad de los dispositivos, que en otros lugares las autoridades de regulación han comenzado a tomar en consideración44, mientras en Europa no han sido afrontados ni siquiera en el curso del proceso de revisión de la plataforma normativa existente, que desde hace tiempo emprendió la Comisión 45 .

Respecto a la regulación de los tests clínicos, la normativa es extremadamente genérica y se limita a una remisión a los principios generales en materia de experimentación humana, quedando de esta manera privada de la especificidad necesaria para acoger la peculiaridad del uso experimental de dispositivos médicos, como una prótesis robótica.

Esta regulación, en definitiva, solo se refiere a los dispositivos de uso médico, es decir, a aquellos dispositivos que tienen un fin terapéutico o de diagnóstico. Sin embargo, según la enunciación de la Directiva 42/93 (art. 2), la tarea de definir la finalidad médica del producto es reenviada al mismo fabricante que solicita la certificación del producto y al material informativo con la cual la acompaña. La razón de esta solución, confirmada en una ocasión por la Corte de Justicia ${ }^{4}$, es la de proteger la salud de los pacientes, pero también la de asegurar la libre circulación de los bienes en el territorio comunitario. Por estas razones, a este régimen podrían escapar aplicaciones robóticas que solo tengan como objetivo el aumento de potencialidades, y no de terapia, aunque presenten características invasivas similares a las de los dispositivos médicos propiamente dichos ${ }^{47}$.

44 FDA, Cybersecurity for Networked Medical Devices Containing Off-the-Shelf (oTS) Software, I4 de enero de 2005 (www.fda.gov/downloads/MedicalDevices/DeviceRegulationandGuidance/ GuidanceDocuments/ucmo77823.pdf); FDA, Content of Premarket Submissions for Management of Cybersecurity in Medical Devices, 2 de octubre de 2014 (www.fda.gov/downloads/MedicalDevices/DeviceRegulationandGuidance/GuidanceDocuments/Ucm356r9o.pdf). Ver, además, el documento Attack Surface: Healthcare and Public Health Sector, emanado recientemente por el National Cybersecurity and Communications Integration Center en el US Department of Homeland Security, que describe las vulnerabilidades asociadas a los dispositivos empleados en el ámbito sanitario (www.kslaw.com/library/publication/HHo5 I4 2 2:Bulletin.pdf).

45 Ello debería concluirse con el dictado de un reglamento que sustituye a las directivas precedentes: European Commission, Proposal for a Regulation of the European Parliament and of the Council on medical devices, and amending Directive $2001 / 83 / E C$, Regulation (EC) No I78/2002 and Regulation (EC) No I223/2009, сом (2012) 542 final, Brussels, 26 de septiembre de 2012.

46 Cgue, Case C-2 I9/I I Brain Products GmbH v BioSemi vof and Others [201 2].

47 En el proceso de revisión de la Directiva este problema ha sido considerado y ha derivado en la propuesta de introducir en la nueva reglamentación un agregado que contiene una lista especial 


\section{A. De la calificación genérica a la laguna normativa}

Si por regla existe una base normativa que ofrece un marco jurídico para muchas de las tecnologías robóticas actuales, lo expuesto pone en evidencia en qué medida pueden existir implementaciones que no recaigan en los regímenes existentes y, por lo tanto, queden por fuera de la regulación.

Un segundo ejemplo, muy claro, es proporcionado por los vehículos autónomos que, desde el punto de vista jurídico, se ubican en un área gris. La circulación en las calles de automóviles sin conductor es posible, actualmente, por reglamentaciones especialmente introducidas para consentir su uso. Una detallada investigación sobre la legislación estadounidense, a nivel federal, incluida la regulación derivada de la Convención de Ginebra de 1949, y de cada uno de los Estados, concluyó que "probablemente", en la actualidad, su uso no está prohibido $^{48}$. No obstante, una intensa actividad de lobbying ha llevado a la emanación de disposiciones ad boc que autorizan la circulación en vía experimental por las calles de diversos Estados (los primeros en legislar sobre la materia han sido Nevada, Florida, California y Michigan). Estos se han ocupado de reglamentar el permiso especial que legitima la circulación de estos vehículos; de imponer algunos requisitos de seguridad, como la presencia a bordo de una persona que pueda asumir el control del vehículo en caso de necesidad; de los perfiles de la responsabilidad en caso de incidente y los aspectos asegurativos ${ }^{49}$.

En Europa, por el contrario, la Convención de Viena sobre el tráfico urbano de 1968 excluye, en forma plausible, la circulación de vehículos autónomos por las vías públicas, comoquiera que una norma impone que cada vehículo debe

de "productos implantables o, incluso, invasivos sin un objetivo médico", que han de considerarse como dispositivos sometidos al mismo régimen, con prescindencia del hecho de que sean definidos para uso médico por el fabricante (art. 2.I). La cuestión es ampliamente discutida en Maslen, H., et al., The regulation of cognitive enhancement devices: Refining Maslen et al.'s model, en fournal of Law and the Biosciences, 2015, I-I 4 y en el artículo precedente de los mismos autores, "The regulation of cognitive enhancement devices: extending the medical model", ibíd., 2014. Vale la pena destacar que la solución descrita, si fuese finalmente acogida, se ocupa únicamente de garanizar estándares mínimos de seguridad del producto, pero no concierne en sí misma a la legitimidad de dispositivos con fines de potencialización.

48 Walker Smith, B., Automated Vehicles Are Probably Legal in the United States, i Texas AઐM Law Review, 20I4, 4I I-52 I. La National Highway Traffic Safety Administration (NHTSA) ha asumido una posición clara a favor del desarrollo de tecnologías de automatización avanzada para el transporte: ver el Preliminary Statement of Policy Concerning Automated Vebicles, y principalmente el sucesivo 20 I 6 Update.

49 Otros aspectos regulados en las leyes de los estados americanos pueden referirse a: la indicación a otros conductores, mediante licencias especiales, de que se trata de vehículos autónomos; el registro de los datos sobre eventuales colisiones y la obligación de transmitirlos a la autoridad competente; las restricciones a la posibilidad de utilizar vehículos autónomos en ciertas partes del territorio. Para un análisis detallado de la legislación, además del trabajo citado en la nota nota 48, espec. 500-508, ver también Gosselin, K. M., Navigating the Policy Landscape to Bring Autonomous Vehicle Legislation to Your State, New York University Fournal of Legislation and Public Policy Quorum, 2015, 85-109, espec. 9I-97. 
estar permantemente bajo el control de un conductor ${ }^{\circ}$. Su formulación no consentiría una interpretación evolucionista, en línea con el objetivo, propio de la misma Convención, de garantizar e incrementar la seguridad en el tráfico, en sustancia, a través de la prohibición de la automatización integral del proceso de conducción. Sin embargo, esta estaría permitida dentro de cierto límite, donde se encuentre asegurada la presencia a bordo de una persona que monitoree constantemente la situación del tráfico y esté en capacidad de asumir el control del vehículo, excluyendo el dispositivo automático ${ }^{5}$.

Un paso preliminar en la transición hacia la automatización, cada vez más avanzada, en el sector del transporte es el de calificar jurídicamente a los vehículos sin conductor como vehículos admitidos en la circulación urbana, a falta de lo cual podría dudarse de la legitimidad de su introducción en el tráfico común. Como consecuencia de lo anterior, en el corto plazo se podría producir su asimilación a los vehículos comunes y, por ende, su sujeción al mismo régimen jurídico, tanto en el plano asegurativo como en el de la responsabilidad. Por el contrario, un mercado más maduro, en el cual cambien los modelos económicos sobre los cuales está establecido el sistema de transporte, debería estar asistido por una mutación de las reglas de responsabilidad, que permita respaldar el objetivo de una mayor seguridad garantizada por el sistema automático de guía y, al mismo tiempo, el resarcimiento íntegro de las víctimas de los (raros pero inevitables) accidentes.

\section{Robot y tutela de los consumidores}

También resulta interesante la perspectiva que examina el mercado emergente de la robótica a la luz del derecho de los consumidores y, en particular, de la disciplina de las prácticas comerciales desleales ${ }^{52}$. De hecho, algunas características intrínsecas de los robots o de las estrategias de marketing perseguidas por las empresas del sector presentarían elementos de fricción en relación con la línea de tolerancia marcada por la tutela en favor de una información comercialmente correcta. En efecto, por un lado, se plantean interrogantes sobre la compatibilidad de los sistemas robóticos, con frecuencia antropomorfos, asiduamente capa-

50 En el texto inglés, el artículo 8 establece que "Every moving vehicle or combination of vehicles shall have a driver" (inc..$^{\circ}$ ), y que "Every driver shall at all times be able to control his vehicle..." (inc. $5 \cdot^{\circ}$ ).

5 I Con posterioridad a una modificación al artículo 8, introducida con ocasión del unece, se acepta sustancialmente la conformidad de los sistemas de asistencia a la conducción con la Convención cuando ellos puedan ser desactivados por el conductor o el conductor pueda asumir el control superando el dispositivo automático: ver Working Party on Road Traffic Safety, Sixtyeighth Session, 24 a 26 de marzo de 2014, Ginebra, Report of the Sixty-eighth Session of the Working Party on Road Traffic Safety (ECE/Trans/WP. I/I45). El nuevo texto entró en vigor el 23 de marzo de 2016.

52 Hartzog, W., Unfair and Deceptive Robots, 74 Maryland Law Review 20 I 5, 785. 
ces de desencadenar sentimientos de empatía y de mostrar inicios de interacción social, con fines de transparencia y ausencia de falsedad en los productos o en las campañas publicitarias que los promueven. En otras palabras, la naturaleza y las características de, por lo menos, algunos de los robots que están o que pronto estarán disponibles en el mercado podrían introducir comportamientos no racionales, como la capacidad de simular, especialmente en los sujetos más vulnerables como los niños o los ancianos, emociones diversas de aquellas que habitualmente se tienen hacia una máquina ${ }^{53}$. Se cuestiona entonces si los robots sociales, que son proyectados para suscitar sentimientos e interacciones con los seres humanos mediante la apariencia de reacciones emotivas, en realidad inexistentes, no son por su naturaleza productos engañosos susceptibles de manipular, especialmente, a las personas más frágiles y menos conscientes ${ }^{54}$. Este tipo de interacción podría ser estimulada, incluso, por un marketing del producto, que no ponga de relieve la naturaleza claramente mecánica del robot o el hecho de estar teleguiado en el movimiento, en el lenguaje y en la gestualidad. Igualmente crítica sería la posible confusión entre la apariencia autómata del robot y el hecho de que en realidad es guiado a distancia por un operador invisible para el usuario, con la consecuencia de que este podría actuar sin saber que está siendo visto y oído.

En segundo lugar, los mismos fabricantes y distribuidores de robots tendrían amplios márgenes para una presentación deshonesta de sus productos, y actualmente ya existen evidencias en este sentido: desde las características que todavía se encuentran en fase experimental, pero son presentadas como si existieran, a la aceleración del movimiento del robot en los videos de presentación, para mejorar en apariencia el desempeño55. En relación con estas prácticas también se plantea la necesidad de un control dirigido a discernir entre las conductas abusivas y las que no exceden los límites de las prácticas comerciales comunes.

Sin embargo, la mayor preocupación nace de una verdadera revolución que las tecnologías robóticas se encontrarían en capacidad de introducir en la dinámica del comercio: gracias a la capacidad de almacenar datos y de asociarlos a través de su conexión a la red, los robots tendrán mucha información sobre nosotros y podrán usarla para hacer ofertas de nuevos productos en tiempo real, o

53 Se da el ejemplo del niño que manifiesta apego afectivo hacia el robot teleoperado remotamente y le confía secretos que no diría a los padres o a los maestros, o bien del robot acompañante que le solicita al propio propietario que adquiera una actualización haciendo movimientos mímicos ante imprevistas dificultades de funcionamiento: Hartzog, Unfair and deceptive robots, cit., 787,804 .

54 Considérese la previsión de la Directiva 2005/29/CEE sobre las prácticas comerciales desleales que se refiere a "commercial practices which are likely to materially distort the economic behaviour only of a clearly identifiable group of consumers who are particularly vulnerable to the practice or the underlying product because of their mental or physical infirmity, age or credulity in a way which the trader could reasonably be expected to foresee" (art. 5 , inc. 3 ).

Hartzog, Unfair and deceptive robots, cit., 793. 
incluso venderlos por su valor. El hecho de que los robots sean objetos tangibles y móviles permite que sean utilizados para recoger datos sobre el consumo y sobre las preferencias individuales, no solo en el ambiente digital, sino también en el mundo real, que luego serán utilizados para realizar estrategias de marketing personalizadas, sin necesidad de que el usuario acceda a la red ${ }^{6}$. En otras palabras, las tecnologías robóticas permiten obtener amplios conocimientos sobre los deseos y los intereses de consumo del público, en un grado mucho mayor al de las estrategias comúnmente usadas en la actualidad (como encuestas telefónicas o mediante internet, elaboración de perfiles de clientes o campañas de fidelización), puesto que la interacción con los clientes y la recopilación y gestión analítica de información no se realizarán únicamente mediante instrumentos telemáticos, sino a través de diversos medios. De esta información será posible realizar un seguimiento, procesarla y combinarla, para luego aplicarla a técnicas de marketing dirigidas a obtener la más elevada tasa de conversión entre la oferta de un producto y su venta efectiva. El cuadro que se va delineando muestra más de un aspecto de divergencia respecto a los cánones de transparencia y de corrección sobre los cuales está basado el mercado de provisión de bienes y servicios a los consumidores. Por lo tanto, la idoneidad de las actuales técnicas de protección del consumidor deberá ser objeto de un constante monitoreo.

Ligada a la tutela de los consumidores en sentido lato, también se encuentra la perspectiva que se cuestiona sobre el vínculo entre la aparición de los robots en ambientes ordinarios y la protección de la privacidad de los datos personales. Los sistemas robóticos están equipados con una red muy sofisticada de sensores, videocámaras, tecnologías para el reconocimiento facial y de los objetos, que permiten percibir al ambiente circundante en un modo bastante preciso y, de hecho, convertirse en instrumentos de vigilancia ${ }^{57}$. Las preocupaciones por la vida privada comprenden tanto a los robots destinados a operar en ambientes domésticos y en íntimo contacto con las personas ${ }^{5}$ como a las tecnologías como aquella de los drones ${ }^{59}$. Estos últimos pueden alcanzar fácilmente lugares generalmente inaccesibles, pueden estar equipados con telecámaras de alta resolución o termo-cámaras para la visión nocturna, además de micrófonos y, en consecuencia, pueden efectuar capturas fotográficas o de comunicaciones. Sus dimensiones pueden ser muy reducidas, al punto de hacer difícil su visualización y señalización. La naturaleza ubicua de la tecnología aumenta su potencial, pero

${ }_{5} 6$ Posibles escenarios son descritos a modo de historias de ciencia ficción por NourbakHsh, Robot tra noi, cit., 23 ss.

57 Calo, R., Robots and Privacy, en Robot Ethics. The Ethical and Social Implications of Robotics, cit., I87-202.

58 Kaminsкi, M. E., Robots in the Home: What Will We Have Agreed to?, Idaho Law Review, 20 I 5, vol. $5 \mathrm{I}, 66 \mathrm{I}-677$.

59 "They represent the cold, technological embodiment of observation": Calo, R., The Drone as Privacy Catalyst, Stanford Law Review online, 20 I I, 64, 29-33, 33. 
es por muchas razones disonante con las modalidades de protección de los datos personales, sobre los que se basa la normativa actual. No por casualidad, el sector de la robótica es uno de los más involucrados por el concepto de privacidad by design implementado también por el nuevo Reglamento sobre el tratamiento de los datos ${ }^{60}$.

\section{Los problemas de la seguridad y de la responsabilidad en el mercado emergente de la robótica}

Garantizar una seguridad elevada de los propios productos y proyectar respuestas adecuadas ante la eventualidad de que causen daño a quien los utiliza o a terceros constituye una de las preocupaciones constantes de la industria robótica. Los sistemas robóticos están cada vez más pensados para ser introducidos en ambientes comunes, en íntima relación con el hombre, circunstancia que aumenta, junto con la versatilidad de la tecnología, las ocasiones de contacto y de impacto accidental. Respecto a los robots industriales -máquinas que son destinadas a espacios predispuestos para acogerlos con barreras físicas y virtuales, y dotadas de dispositivos que se detienen o reducen su movimiento en caso de que una persona ingrese al área limitada- el riesgo de accidente está contenido. Mucho más difícil es introducir sistemas de seguridad confiables en relación con los robots móviles, que son diseñados para circular en ambientes no estructurales, como el tráfico urbano ordinario, o para ser introducidos en viviendas privadas, o para actuar en íntima relación con una persona que sufre discapacidad o que necesita de una terapia de rehabilitación.

Es el caso de los robots asistentes, que deben moverse en ambientes altamente dinámicos y unir autonomía y flexibilidad para asistir al individuo en las actividades cotidianas; el sistema de sensores con los que son dotados debería permitir su localización, evitar los obstáculos, planificar la trayectoria a seguir, pero la complejidad de estas operaciones es tal que no permite eliminar totalmente los riesgos que ellas comportan. Todavía más evidente es el problema relativo a los vehículos autónomos, que según algunas estimaciones pueden aumentar notablemente la seguridad del tráfico en la calle, dado que no sufren de déficit de atención, de problemas de distracción, de imprudencia o impericia; en efecto, su software se encuentra en capacidad de registrar y seguir la trayectoria de varios objetos en movimiento a lo largo de un campo visual más amplio que el humano y cuentan con tiempos de reacción más rápidos. Sin embargo, el riesgo principal está determinado por la necesidad de compartir el ambiente operativo con otros

60 Reglamento 2016/679 del Parlamento europeo y del Consejo del 27 de abril de 20i6, relativo a la protección de las personas físicas en lo que respecta al tratamiento de datos personales y a la libre circulación de estos datos, y que deriva de la Directiva 95/46/CE. Ver, en particular, art. 25 . 
vehículos normalmente guiados y con peatones cuyo comportamiento puede ser imprevisto, así como por la velocidad de funcionamiento que no puede ser limitada en forma excesiva, so pena de privarlos de utilidad para el desplazamiento. Por otra parte, los robots que se utilizan para la rehabilitación de las personas, como por ejemplo las extremidades que se utilizan después de un ictus cerebral o de una lesión de la médula espinal ${ }^{61}$, dispositivos que se llevan consigo y que por lo tanto están destinados a ser utilizados en estrecho contacto con el cuerpo, suscitan preocupaciones respecto a la seguridad de la persona que los usa. Por su parte, sistemas como las prótesis biónicas o las interfaces cerebro-máquina que maniobran una silla de ruedas u otra plataforma robótica plantean el problema relativo a la correcta interpretación de la señal que conduce el movimiento del miembro artificial o del dispositivo externo.

Las tipologías de riesgos a afrontar se encuentran ligadas a los vicios de ingeniería o de programación (un escaso examen de los componentes mecánicos, defectos del sistema electrónico o del software, algoritmos no confiables), a errores humanos del operador, a condiciones ambientales que obstaculizan la actividad de los sensores. Un mal funcionamiento podría generar que el robot no se detenga ante la presencia de un obstáculo, que asuma una velocidad excesiva, que se ponga bruscamente en movimiento, en definitiva, que asigne una respuesta incorrecta a los estímulos que recibe ${ }^{62}$. Pero existen otros tipos de riesgos a tener en consideración, como el de la seguridad del sistema informático contra potenciales ataques externos, que envían al robot comandos no autorizados y dañan el sistema operativo ${ }^{6}$. $^{6}$.

Estas consideraciones esbozan, sumariamente, las razones de orden técnico y socioeconómico que hacen crucial el tema de la seguridad y de la responsabilidad en el análisis jurídico de la robótica. La preocupación por la fijación de estándares elevados de seguridad y por una clara definición de las reglas sobre la atribución de la responsabilidad depende principalmente de las características de la robótica de servicio, destinada a generar formas de íntima interacción hombre-máquina, y con ello a multiplicar en forma exponencial las ocasiones de daño. Por su parte, las delicadas tareas que podrían ser destinadas a un robot y la cercanía con usuarios vulnerables como los enfermos, personas ancianas o

6I Como el Lokomat, sobre la cual véase la investigación de RiEnER, R. et al., Locomotor Training in Subjects with Sensori-Motor Deficits: An Overview of the Robotic Gait Orthosis Lokomat, Fournal of Healthcare Engineering, 2010, I, I97-2 I6.

62 Vasic, M. y Billard, A. Safety Issues in Human-Robot Interactions, en Proceedings of the 2013 IEEE-RAs International Conference on Robotics and Automation, Karlsruhe, 6 a Io de mayo de 2013 .

63 Lo subraya, junto con los demás problemas de seguridad a considerar en la gestión de los riesgos, Wu, S. S., Risk Management in Commercializing Robotics, 3 de abril, 201 3, en: http://conferences.law.stanford.edu/werobot/wp-content/uploads/sites/29/2013/o4/Risk-Managementin-Commercializing-Robotics.pdf 
con discapacidad, aumenta el riesgo de que provoquen lesiones graves en caso de accidente. Pero sobre todo, las características del mercado emergente de la robótica hacen predominantes las exigencias de seguridad frente al riesgo de su salida al mercado, en caso de que se presenten controversias por daños del producto. En otras palabras, se vuelve a plantear una situación típica de la inserción de innovaciones tecnológicas que hoy ya son comunes, como la aviación civil en el sector de los aviones pequeños y de sus componentes, que han corrido el riesgo de desaparecer por efecto de los altos costos (estimados y en parte soportados) de las acciones civiles por daños ${ }^{64}$. Otra similitud se verifica con el internet, que logró expandirse gracias a la exoneración sustancial de la responsabilidad de los web providers por los contenidos ofensivos o lesivos de derechos de privacidad ajena, difundidos mediante la web ${ }^{65}$.

Junto con estos elementos existen razones técnicas, relativas a la peculiar naturaleza de algunas implementaciones robóticas, que justifican la centralidad del tema de la responsabilidad. Se alude a la creciente capacidad de las máquinas -se trate de vehículos que circulan por las calles o de robots asistentes utilizados en la propia casa, en un contexto hospitalario, o en una casa de asilo de ancianos- de funcionar y moverse sin conducción humana; característica que, dando lugar a la apariencia de autonomía, parece sustraer la acción de los robots de la esfera de control de la persona. A esto obedece la dificultad de encuadrar la responsabilidad derivada de los accidentes producidos por el uso de la tecnología robótica en un orden conceptual que normalmente presupone la correspondencia entre la posibilidad de control, la capacidad de evitar la verificación de un daño y la relativa imputación de la responsabilidad. En su forma más débil, esta secuencia lógica exterioriza un nexo de causalidad entre conducta/omisión del agente y evento dañoso; y en la hipótesis de la responsabilidad por culpa pone de relieve el reproche a la subjetividad del agente.

Los robots están en capacidad de actuar sobre el ambiente que los rodea prescindiendo de una guía, en consecuencia, sin ser tele-operados; los productos más evolucionados podrán incluso moverse o cumplir acciones de manera independiente, mediante instrucciones escritas ex ante en su sistema operativo. La autonomía, en sus diversos grados y extensiones, es considerada una característica típica de los robots, que es necesario potenciar y mejorar si se pretende que

64 Ver Calo, R., Open Robotics, 70 Maryland Law Review 20 I I, I32, y la referencia a la General Aviation Revitalization Act (GARA) emanada en el año I994 en Estados Unidos justamente para remediar la crisis de la industria de los viajes de placer. Sobre esta regulación ver también ScoTт, D. S., Note, "The General Aviation Revitalization Act of r 994: The Initial Necessity for, Outright Success of, and Continued Need for the Act to Maintain American General Aviation Predominance Throughout the World", 34 Oklaboma City University Law Review 2009, 75.

65 Esta analogía es desarrollada por CALo, Open Robotics, cit., I33 s., de manera conjunta con la relativa a la inmunidad establecida en Estados Unidos para los fabricantes de armas de fuego, respecto de los ilícitos cometidos con ellas por sus adquirentes. 
estén en capacidad de actuar en ambientes complejos y responder a los estímulos externos de manera apropiada. En efecto, una estructura abierta los hace capaces de reaccionar de manera eficiente, y no necesariamente predeterminada, a los inputs que reciben. La capacidad que poseen de aprender de la experiencia hace de su capacidad de reacción algo de cierta forma imprevisible, toda vez que podrían mostrar "comportamientos emergentes" ${ }^{66}$, es decir, no previstos en la fase de proyección, sino fruto de interacciones inesperadas entre los componentes del sistema o con el contexto en el que operan. La multiplicidad de usos a los que podría estar destinado un robot, y los contextos en los que estaría llamado a operar, difieren entre sí, al punto que no pueden ser anticipados por su propio diseñador y programador, a efectos de adoptar las precauciones y previsiones necesarias ${ }^{67}$. Además, los usuarios podrían interactuar con los robots en la medida en que ellos funcionen como plataformas abiertas (es decir, creados para un uso no específico, que utilizan un software open source y presenten un diseño modular), que podrían ser manipuladas por terceros en manera no prevista por el fabricante ${ }^{68}$.

De tiempo atrás se ha puesto en evidencia la manera como la tecnología contribuye a aumentar la complejidad de los sistemas, a volver inevitables los errores, así como a aumentar la incertidumbre en cuanto a la secuencia causal que los provoca y a la imputación subjetiva. En el caso de la robótica, que conjuga artefactos de ingeniería con software y sistemas de control artificiales, e introduce en ellos conocimiento y capacidad de decisión ${ }^{69}$, este fenómeno toma mayor relevancia ${ }^{70}$. En la puesta en funcionamiento de sistemas robóticos complejos, los roles y las competencias de diversas personas se superponen y están íntimamente interconectados; y quien contribuye en un segmento de la operación conjunta, con frecuencia no tiene el control sobre la misma, y a veces, incluso, tampoco conoce la estructura ni comprende el funcionamiento del dispositivo en su totalidad. La capacidad de interacción y de manipulación del ambiente y la

66 Arkin, R. C., Behavior-based Robotics, Cambridge (Mass.), i 998.

67 Asaro, P., Robots and Responsibility from a Legal Perspective, 2007, 2 (manuscrito inédito disponible en: http://www.peterasaro.org/writing/ASARO\% 20Legal\% 20 Perspective.pdf).

68 Esta constatación explica la propuesta regulatoria de CALo, Open Robotics, cit.

69 El término inglés "cognition" se refiere a "la capacidad de decisión en la inteligencia artificial y los procesos de control de un robot empleado para elegir de qué manera interpretar los inputs de sus sensores, cómo atribuir un significado a los mismos en un contexto determinado y, finalmente, cómo elegir qué hacer": según la definición del glosario elaborado por NourbakHsh, Robot fra noi, cit., I49.

70 Von Schomberg, ob. cit., 333; Id., Robot Ethics: Mapping the issues for a mechanized world, en Artificial Intelligence, 20 I I, I 75 (5-6), 942-49; WALLACH, From Robots, cit., I 94. Que la responsabilidad sea considerada la principal preocupación es evidente desde los primeros estudios generales acerca de las implicaciones éticas, legales y sociales de la robótica: BeKEy, G., Lin, P. y Aвney, K., Ethical Implications of Intelligent Robots, en Neuromorphic and Brain-Based Robots, J. L. Krichmar y Wagatsuma, H. (eds.), Cambridge-New York, 20 I I, 323 ss. 
imprevisibilidad de los comportamientos crearían una fricción con el fundamento común de las reglas de responsabilidad por daños y con los modos tradicionales de los cuales el sistema jurídico se vale para atribuirla, basados en la culpa y en la relación de causalidad ${ }^{71}$. De la dificultad para asignar la responsabilidad en los sistemas complejos, pero principalmente de la supuesta incompatibilidad entre la ratio de este tipo de reglas y el modo de operación de los productos robóticos, se desprende la necesidad de romper con los modelos existentes y razonar según esquemas innovativos.

\section{A. Las propuestas de esquemas alternativos a las reglas comunes de la responsabilidad}

Mientras los diversos análisis comparten la premisa acerca de las características especiales que detentan los sistemas robóticos, proyectan soluciones diversas para afrontar el llamado "responsibility gap". Las principales propuestas pueden articularse en una triple partición.

Una primera corriente confiaría al instituto de la limitación de la responsabilidad una doble tarea: por un lado, la de promover la innovación en la investigación y en la industria robótica, reduciendo el temor ante elevados costos ligados a la participación en las controversias civiles; por el otro, la de garantizar inmunidad a los fabricantes frente a eventos dañosos que no habrían podido ser evitados usando la debida diligencia, al diseñar el producto y al informar al consumidor de sus riesgos potenciales. Esta idea de una "inmunidad selectiva", que se aplicaría principalmente a los fabricantes de esquemas robóticos abiertos, constituiría un compromiso eficiente con la necesidad de dar impulso al desarrollo de tecnologías innovativas y la de incentivar la adopción de medidas de seguridad ${ }^{72}$.

Una segunda tesis recurre a la creación de una personalidad jurídica para los robots a los fines de convertirlos directamente en responsables de los eventuales daños causados a terceros ${ }^{73}$. Esta posibilidad es considerada más eficiente respecto al intento de adaptar los actuales esquemas de responsabilidad tradicional a las hipótesis de los robots con autonomía limitada. Estos esquemas podrían encontrar aplicación en los casos en los que se considerara asimilable la capacidad cognitiva y decisoria de los robots a la de los sujetos que, por la edad o la debilidad en el plano psíquico, no están obligados a responder en primera per-

7 I Matthias, A., The responsibility gap: Ascribing responsibility for the actions of learning automata, en Ethics and Information Technology, 2004, 6, I 75-183.

72 CaLo, Open Robotics, cit., I 3 I ss.

73 Leroux, C. et al., Suggestion for a green paper on legal issues in robotics. Contribution to Deliverable D3.2.I on ELS issues in robotics, disponible en: http://www.eurobotics.net/cms/upload/PDF/euRobotics_Deliverable_D.3.2.I_Annex_Suggestion_GreenPaper_ELS_IssuesInRobotics.pdf 
sona por los daños generados a otros, y son sustituidos en la función resarcitoria por aquellos sujetos que los tienen a cargo. Igualmente, y tal vez en modo más apropiado, la acción podría compararse con la de seres dotados de una racionalidad primitiva, como la de los animales, la cual involucra la responsabilidad de su propietario. Sin embargo, esta responsabilidad por defecto en el control de la actuación del robot podría imponer cargas excesivas al propietario, en los casos en que la propia tecnología es usada con el fin de aliviar la condición de las personas que, como los ancianos o los discapacitados, los utilizan con fines de asistencia y cuidado. En estos casos se vería frustrada la razón de ser de la introducción de estas implementaciones tecnológicas, es decir, hacer accesible a más personas la disponibilidad de una ayuda doméstica, en respuesta a los problemas de envejecimiento de la sociedad, de la escasez de asistencia de tipo profesional y de los costos elevados que la misma representa, así como de la necesidad de promover la independencia y la inclusión social de sus usuarios potenciales.

A partir de la capacidad embrionaria -aunque probablemente en aumentode los robots, de expresar un alto nivel de autonomía, se propone un argumento más general dirigido a construir una subjetividad del robot74. La "personalidad electrónica" es considerada un enfoque plausible al problema de la responsabilidad, tanto para los robots dotados de un cuerpo como para los softwares robots que exhiben un cierto grado de autonomía e interactúan con las personas. A esta propuesta se suma, naturalmente, la necesidad de crear un registro y dotar a cada robot de una identificación al momento de su puesta en el comercio, además de asegurar que le sea asociado un fondo a través del cual sea factible responder por las obligaciones. Los mecanismos por medio de los cuales se podría formar y financiar este fondo podrían ser diversos, y la respectiva elección implicaría la identificación del sujeto sobre el cual deberían recaer, en todo o en parte, las consecuencias económicas de los eventuales daños provocados por la máquina ${ }^{75}$.

Una tercera indicación se encuentra orientada en sentido opuesto, es decir, hacia un incremento de la responsabilidad del propietario de la máquina frente a la tutela del eventual perjudicado $7^{7}$. La constatación de la que se parte es la dificultad para este último de probar la negligencia del propietario, o bien, en el caso de que opere un régimen de responsabilidad por producto defectuoso, el defecto del producto y el nexo de causalidad, en razón a la complejidad y al carácter "inescrutable" del funcionamiento de máquinas extremadamente sofisticadas para una persona común. Por el contrario, el propietario debería responder con base en un criterio de responsabilidad objetiva, dado que como beneficiario de la

74 Leroux et al., ob. cit., $5^{8}$ ss.

75 Explora esta hipótesis de encuadramiento del problema de la responsabilidad también el Draft Report del IURI Committee, punto 3I.f).

76 Decker, M., Responsible Innovation for Adaptive Robots, en Retbinking Responsibility in Science and Technology, Battaglia, F., Mukerji, N. y Nida-Rümelin, J. (eds.), Pisa, 20i4, 65 ss. 
tecnología puede obtener ventajas económicas y productivas mediante la introducción del robot en su organización. Puesto que esta regla debe ser sostenible y compatible con el avance del proceso de automatización y con la difusión de la robótica de servicio, se propone acompañarla con la fijación de un límite máximo de resarcimiento al cual podría estar sujeta la misma persona, circunstancia que permitiría asegurar el riesgo con mayor facilidad.

\section{B. Algunas observaciones críticas}

La variedad de las propuestas delineadas resalta la centralidad del tema de la responsabilidad en la reflexión jurídica sobre la tecnología y, en particular, sobre la robótica. Dentro de su diversidad, ellas comparten un tramo común, en el sentido de que parten del presupuesto de una novedad radical en las características del agente material y en la causación del evento dañoso, al punto de imponer una salida del régimen común de imputación de la responsabilidad. Sin embargo, esta concepción no parece plenamente convincente y las soluciones operativas a las que conduce son discutibles.

La idea de exonerar a los fabricantes de tecnologías robóticas de la responsabilidad por eventos que, aunque atribuibles causalmente a la acción de un robot, quedan efectivamente fuera de su capacidad de control, se conectan con el objetivo de favorecer el aumento de la industria del sector. De hecho, los fabricantes del sector difícilmente estarían en capacidad de anticipar y, por lo tanto, de internalizar los costos, potencialmente elevados, de las controversias civiles para el resarcimiento de los daños causados. Se asume evidentemente que la robótica representa un caso especial, en el cual a la deseabilidad social de la innovación en el sector se suma la presencia de riesgos constantes y difícilmente determinables con antelación, ligados a las características propias de la tecnología. Sin embargo, no son claras las razones que justifican este tratamiento, y el régimen especial parecería aplicable a cualquier tipo de esquema robótico por el hecho de compartir las mismas características técnicas, sin distinguir en razón a la mayor o menor capacidad de aplicaciones singulares para dar respuestas a las necesidades sociales cualificadas.

Esta propuesta realmente madura en el sistema estadounidense, en el cual se adopta un enfoque que dista del europeo en cuanto a la regulación de la tecnología. En efecto, mientras en el contexto europeo está fuertemente radicado el principio de precaución, que presenta numerosas bases normativas y se ha extendido de la tutela al ambiente a otros sectores como el de la biotecnología, en Estados Unidos es más común un tratamiento en términos de evaluación del riesgo. Por lo tanto, una menor importancia adquieren las consideraciones relativas a la apreciación, en el plano social, de ciertos progresos científicos y tecnológicos, en la óptica de un equilibrio con los riesgos aceptables. Cabe plantearse, igualmente, si esta solución, además de ser poco convincente en términos 
generales, no sea incluso inútil, en la medida en que se refiera exclusivamente a los productos manipulados después de su adquisición. De hecho, tanto en Europa como en Estados Unidos, la responsabilidad por producto defectuoso, de la que se querría sustraer a las aplicaciones robóticas, se aplica a los defectos originarios, y bien podría ser excluida en los casos en que la acción dañosa tenga relación con modificaciones introducidas al dispositivo en un momento sucesivo a su comercialización en el mercado gracias al carácter abierto de su sistema operativo.

La tesis desarrollada en el ámbito del grupo de investigación agregado en torno al proyecto euRobotics también se expone a algunas dudas en relación con dos aspectos específicos. El primero respecta a la posible confusión o interpretación errónea ínsita a la creación de una personalidad jurídica para los robots. Claramente esta es entendida únicamente en un sentido funcional, es decir, como mecanismo que permite la imputación de efectos directos sobre la máquina, para que de esta manera se puedan repartir las cargas económicas del resarcimiento entre los sujetos que están comprometidos en el financiamiento del patrimonio que está involucrado, sin hacerla recaer sobre uno solo de ellos. De hecho, una responsabilidad exclusiva y no distributiva tendría repercusiones negativas de diversa naturaleza. La prospectiva de afirmar una responsabilidad del fabricante, sujeto que por lo general cuenta con una mayor solvencia $-y$ por lo tanto es comúnmente elegido por el perjudicado para ser demandado en la acción de resarcimiento-, tendría un efecto desincentivante en el desarrollo de tecnologías innovativas, pero no exentas de riesgos. Además determinaría un aumento de los costos de aplicación de tecnologías que tienen utilidad social, tornándolas menos accesibles a todos los potenciales interesados. Por otra parte, el propietario o el usuario de estas nuevas tecnologías podría no garantizar en manera eficiente el objetivo igualmente importante de la compensación; o bien debería recurrir a mecanismos asegurativos que de nuevo podrían incidir negativamente en la accesibilidad a la tecnología, en especial en la fase inicial de su difusión.

Aunque estas sean las razones técnico-jurídicas que sirven de fundamento a una propuesta como esta, el riesgo de incurrir en una equivocación al atribuir subjetividad a un ente mecánico es muy concreto; es este el de aproximarse a las corrientes que trazan similitudes entre los seres humanos y los robots, con base en las capacidades cognitivas que estos últimos presentan, a fin de afirmar la necesidad de un reconocimiento de su subjetividad ontológica, en la medida en que se acerquen a un estadio intelectual superior ${ }^{77}$. Esta propuesta se pone en

77 Los primeros estudios sobre la subjetividad de los agentes artificiales se remontan a LEHMANWilzig, S. N., Frankenstein Unbound: Towards a Legal Definition of Artificial Intelligence, en Futures, I98 I, I3(6), 442; y Solum, L. B., Legal Personhood for Artificial Intelligences, 70 North Carolina Law Rev. I992, I 23 I. Ver, sucesivamente, Calverley, J. D., Imagining a nonbiological machine as a legal person, en Artificial Intelligence \& Society, 2008, 22; Coeckelbergh, M., Robot rights? Towards 
línea con el pensamiento que se inclina hacia una ampliación de la esfera de los sujetos, para extenderla paulatinamente a los animales $7^{8}$ en cuanto seres sensibles, a los árboles o, en forma más general, al ambiente natural 79 o, justamente, a las máquinas. Que la creación de una inteligencia artificial comparable a la racionalidad humana sea un objetivo alcanzable por la ciencia moderna es puesto en duda por varios de sus propios cultores, tanto en el campo de las neurociencias como en el de la ingeniería y de las ciencias computacionales ${ }^{8 \circ}$; proceder al reconocimiento en el plano jurídico de una nueva categoría de sujetos sería sin duda una elección prematura. Con la ambigüedad de una propuesta dirigida a la atribución a los robots de una personalidad jurídica, se valora la idea planteada en relación con el problema más limitado de la responsabilidad.

Pero más allá del riesgo de incurrir en equivocaciones, se pone en evidencia una segunda crítica, es decir, la redundancia del medio frente al fin. De hecho, con modalidades diversas a la construcción de una personalidad jurídica, la cual parecería excesiva frente al objetivo que se pretende conseguir, podría lograrse el mismo efecto de segregación ${ }^{8}$.

En realidad, la razón de ser de la atribución de la capacidad jurídica podría relacionarse con un objetivo paralelo: esto es, el rol de asistencia personal que los robots están aparentemente destinados a desarrollar podría implicar, en un futuro próximo, asumir obligaciones y realizar operaciones contractuales, principalmente en el contexto de intercambios instantáneos ${ }^{82}$, sin la participación directa de su usuario. De todos modos, la forma jurídica adoptada parecería igualmente exorbitante, puesto que el robot podría ser asimilado a un medio mecánico de transmisión de la voluntad y funcionar como mero intermediario frente al otro contrayente.

a social-relational justification of moral consideration, en Ethics and Information Technology, 2010, I2, 209-2 2 I; Koops, B. J., Hildebrandt, M. y Jaquet-Chiffelle, D.-O., Bridging the Accountability Gap: Rights for New Entities in the Information Society?, Minnesota Journal of Law, Scinece \& Technology, 20IO, I I (2), 508 ss.; Teubner, G., Rights of Non-bumans? Electronic Agents and Animals as New Actors in Politics and Law, 2006, 33(4), 497-52 I; Bensoussan, A., "Plaidoyer pour un droit des robots: de la 'personne morale' à la 'personne robot'", en La lettre des juristes d'affairs, $\mathrm{n} .{ }^{\circ}$ I I34, 28 de octubre de 2013 .

78 Sobre el tema ver Rescigno, F., I diritti degli animali. Da res a soggetti, Turín, 2005.

79 Stones, C. D., Should Trees Have Standing? Toward Legal Rights for Natural Objects, 45 Southern California Law Review 1972, 450.

80 El estado del arte en la materia y las prospectivas futuras son discutidas por Bostrom, N., Super Intelligence. Paths, Dangers, Strategies, Oxford, 20 i4, y en Blackford, R. y Broderick, D. (eds.), Intelligence Unbound: The Future of Uploaded and Machine Minds, Chichester, 20I4.

8I En forma similar, y en sentido fuertemente crítico, recientemente, LoIsEAU, G. y Bourgeors, M., Du robot en droit à un droit de robots, en Semaine juridique, 2014, 2 I64 s.

82 Para especulaciones atinadas en este sentido ver Stradella, E. et al., Robot Companions as CaseScenario for Assessing the 'Subjectivity' of Autonomous Agents. Some Philosophical and Legal Remarks, en Proceedings of the Ist Workshop on Rights and Duties of Autonomous Agents (RDA2) 2OI 2, 29 s. 
Finalmente, la hipótesis de atribuir subjetividad jurídica a los robots parece particularmente inadecuada cuando las tareas de la máquina implican adoptar roles de auxilio a la persona en la vida cotidiana, aunque estos sean llevados a cabo en forma elemental. De hecho, la ventaja de convertir a agentes artificiales en personas jurídicas, dotadas de autonomía patrimonial, se aprecia eventualmente en el plano económico, cuando las acciones y las transacciones a realizar son suficientemente automatizadas, no requieren el ejercicio de la discrecionalidad y no involucran juicios éticos. En este caso, la sustitución de una persona por un ente virtual puede representar un ahorro económico, y justificar de esa forma la ficción jurídica. Esta sustitución, por el contrario, no puede suceder y no debería ser estimulada mediante mecanismos de separación patrimonial, cuando la función del robot sea de asistencia material a personas en situación de vulnerabilidad, para evitar el riesgo de confiarles tareas de cuidado en un sentido más elevado, hasta tanto la técnica progrese al punto de volverlo posible ${ }^{8}$.

El tercer planteamiento, de carácter opuesto a los precedentes, se enfoca exclusivamente en el perfil de la responsabilidad del usuario o propietario del robot, que incluso se quiere convertir en objetiva para favorecer la posición del perjudicado. Esta tesis también parece criticable por dos razones. Por un lado, porque no tiene en cuenta el canal alternativo de la responsabilidad del fabricante. Se trata de la vía que verosímilmente sería tomada con mayor frecuencia por las víctimas potenciales de accidentes, tanto por la mayor solvencia de las compañías fabricantes frente al comprador individual del producto, si se trata de una persona física, como por el régimen actualmente aplicable, que ya facilita la posición del actor. La existencia misma de esta alternativa le resta utilidad a la propuesta de endurecer la responsabilidad del propietario, especialmente cuando ella se basa, esencialmente, en el intento de asegurar al demandado un remedio válido y no excesivamente oneroso. En segundo lugar, porque este planteamiento implicaría endurecer el régimen aplicable a los usuarios finales de las máquinas, que como las víctimas de accidentes podrían pertenecer a una categoría de sujetos vulnerables, como los ancianos o personas con discapacidad para cuya satisfacción de necesidades fue precisamente concebida la tecnología. Esta solución, entonces, además de no parecer dotada de particular eficacia frente al objetivo de garantizar con certeza la compensación, en relación con los propietarios responsables constituiría un elemento disuasorio respecto de la adquisición de la máquina, al punto de desalentar la difusión de una tecnología socialmente

83 DARLING, K. (Extending legal protection to social robots: The effects of anthropomorphism, empathy, and violent behaviour towards robotic objects, en Calo, R.; Froomkin, A. M. y Kerr, I. (eds.), Robot Law, Cheltenham-Northampton, 20I6, 2 I 3 ss.), por el contrario, discute la posibilidad de extender derechos a los social robots, no por una dignidad intrínseca de los mismos, sino por reflejar sentimientos que estamos orientados a proyectar sobre máquinas que exhiben comportamientos sociales y reacciones emotivas. La autora plantea la idea de que esta inclinación natural podría requerir una cierta forma de protección jurídica distinta a la de tipo propietario. 
útil. Parece obvio que la premisa prácticamente tácita de este razonamiento sea el empleo de los robots en el ámbito de una organización empresarial, con exclusión del consumidor individual que no tendría modo de internalizar los costos ligados a acciones de responsabilidad y debería estar especialmente protegido frente a esta eventualidad, principalmente si se encuentra en condiciones de fragilidad personal o económica. Pero el simple hecho de vincular una responsabilidad objetiva a la propiedad de la máquina no consentiría articular la distinción, por el contrario relevante, entre el mero uso personal para fines privados y el uso sistemático y organizado al interior de dinámicas comerciales ${ }^{84}$.

\section{Escenarios presentes y futuros}

Más allá de las críticas que se han generado por la especificidad de las propuestas individuales, es necesario rechazar la idea general que se inclina por el abandono de la disciplina común de la responsabilidad civil, en consideración a las características irreductibles de autonomía e imprevisibilidad de los robots. Las razones son de tipo técnico y de principio. Una primera objeción está relacionada con la especificación de la capacidad de controlar y de internalizar las consecuencias de las propias acciones, como elementos que subyacen a las reglas de la responsabilidad. Para la tesis que se comenta, estos elementos -destinados a desaparecer en un futuro con el uso de máquinas inteligentes, en capacidad de exhibir comportamientos imprevistos y no programados- no justificarían la aplicación de las reglas generales de la responsabilidad al sujeto al cual estarían formalmente dirigidas las acciones del robot. Sin embargo, la realidad es que la ratio subyacente a la imputación de la responsabilidad extracontractual no está unívoca e indisolublemente vinculada a la posibilidad de controlar la actuación propia o ajena. En los sistemas actuales, los criterios de imputación de la responsabilidad son diversos y no todos se encuentran vinculados a esta lógica sobre la cual se fundamenta la regla general de la responsabilidad por culpa ${ }^{85}$. De ello se desprende que, aunque la intermediación de una máquina disminuya, o incluso disuelva, el vínculo existente entre quien la puso en funcionamiento (fabricándola, programándola o haciendo uso de ella) y las consecuencias de su actividad concreta, pueden subsistir suficientes razones para encauzar desde el punto de vista jurídico dichas consecuencias al autor originario. El concepto de riesgo de empresa, de actividad peligrosa, de defectuosidad y de la expectativa razonable de seguridad, incluso el de la culpa, en sus aspectos de previsibilidad y de deber

84 A tal distinción podría corresponder la de robótica industrial y robótica de servicio y, dentro de esta última, la subdivisión en dos grandes segmentos de mercado, la de los professional service robots y la de los personal service robots.

85 Para una síntesis ver Fabre-Magnan, M., Droit des obligations, 2 - Responsabilité civile et quasicontracts, $3 \cdot{ }^{\mathrm{a}}$ ed., Paris, 2013, 62 ss. 
de evitar el daño, siguen teniendo sentido -y por lo tanto siguen teniendo poder para decidir sobre la distribución de los costos de los accidentes- aun en el caso en el que la conducta material es realizada por un agente impersonal, sin que sea invalidada la racionalidad del sistema.

Una segunda objeción, esta vez más general y de principio, concierne a la misma idea de proponer una "fuga" del sistema general de la responsabilidad, sobre la base de la incapacidad de controlar el trabajo de los robots, cada día más complejos y sofisticados, que actúan de manera autónoma e imprevisible. Esta condición, si fuera efectivamente verificable, podría convertirse, de un lado, en el límite mismo de la técnica robótica, puesto que cabría preguntarse acerca de la legitimidad de la construcción de máquinas similares. De otro lado, esa no debería ser la premisa fáctica para no responsabilizar al hombre de la construcción y del uso de los robots; al contrario, la responsabilidad humana, aunque no de manera completamente sobrepuesta a la existente, debe aumentar progresivamente a medida que crece la capacidad técnica para introducir nuevas fuentes potenciales de riesgo en el ambiente. En un interesante ejercicio de autorregulación realizado por un grupo de estudiosos de diversas disciplinas, constituido como Ad Hoc Committee on Responsible Computing ${ }^{86}$, se ha tomado una posición en este sentido, aunque referida a la responsabilidad moral vinculada a la fabricación y al uso de los robots. Una de las reglas acuñadas por el grupo se refiere, justamente, a la eventualidad de que los comportamientos futuros del producto no sean razonablemente previsibles, de la cual se extrae la conclusión de que esto debe implicar un aumento de la responsabilidad de quien lo ha diseñado, de frente a resultados dañosos. La característica de las máquinas robóticas de adaptarse al ambiente con el curso del tiempo, la capacidad de aprender o de auto modificar su propio programa carga a los fabricantes de una mayor responsabilidad en la anticipación de eventuales problemas y en la introducción de medidas preventivas.

Esta admisible constatación, desarrollada en relación con la responsabilidad en el plano ético, no está exenta de encontrar correspondencia en el plano jurídico. El uso de disposiciones conceptuales y de reglas amplias que se refieren a los deberes de información, a la previsibilidad del daño, a los risk management (mediante la aplicación de estándares de seguridad preventivos y el uso de instrumentos de seguridad) para quienes emprenden profesionalmente una actividad que resulta fuente de daños potenciales, permite mantener nuevos y complejos sistemas tecnológicos en la órbita del actual sistema de responsabilidad civil. La utilidad de los robots del futuro está representada, precisamente, por su versatilidad, es decir, por la capacidad de autogenerar el propio programa operativo.

86 Ver la rule 2 en la versión más reciente del documento Moral Responsibility for Computing Artifacts: Five Rules, Version 27, disponible en: https://edocs.uis.edu/kmill/2/www/TheRules/moralResponsibilityForComputerArtifactsV 27.pdf 
Desde el punto de vista técnico, el robot dotado de habilidad de aprendizaje, después de un cierto período en el cual es usado, pasa a ser distinto de los otros robots con los cuales compartió su diseño y fabricación ${ }^{87}$. Sin embargo, toda vez que las características que hacen posible dicho distanciamiento del estándar inicial han sido instaladas en la máquina por el fabricante y por el programador, en el plano jurídico se mantiene intacta la posibilidad de asignar a estos la responsabilidad por los daños eventuales causados.

Es posible, sin embargo, que la evolución en el campo de la robótica y de la inteligencia artificial alcance niveles tales como para requerir, junto con otros factores, la adopción de esquemas diversos de gestión de riesgos y de asignación de responsabilidad, que sean más racionales y eficientes. Podría suceder que el objetivo de la automatización completa, con exclusión total de la participación del hombre en una determinada actividad, sea particularmente deseable en cuanto contribuiría de mejor manera con los objetivos de aumentar la seguridad y de proteger la salud y el ambiente. A su vez, puede ocurrir que para lograr tales objetivos la capacidad de los sistemas de operar por fuera del ámbito predefinido sea esencial, porque garantiza respuestas eficientes a problemas que no pueden ser, en su totalidad, razonablemente anticipados por el fabricante y por el programador. De modo que si se verifica un accidente en condiciones de una efectiva y real autonomía del robot, el mismo no pueda atribuirse al fabricante o al programador, aunque estos hayan generado la capacidad de la máquina de operar autónomamente.

Esto se justifica por varias razones. Principalmente, porque en muchos casos sería difícil identificar una defectuosidad del producto como nosotros la entendemos (es decir, como desviación del estándar de los productos de la misma categoría, o bien como falta de implementación del diseño alternativo "razonable" idóneo para evitar incidentes); por el contrario, es probable que la razón de la reacción que ha determinado el accidente sea indescifrable ${ }^{88}$, al punto de no poder ni siquiera ajustar las versiones sucesivas del mismo producto. En segundo lugar, la carga de la prueba, teniendo en cuenta el test que debe practicarse, sería de extrema complejidad para la víctima hipotética.

Pero sobre todo, la imputación de la responsabilidad al fabricante/programador sería contraproducente en relación con el objetivo de permitir que una

87 Karnow, C., The application of traditional tort theory to embodied macbine intelligence, p. I4, presentado en la We Robot Conference 2013, Stanford Law School, http://works.bepress.com/ curtis_karnow/9

88 VLADECK, D. C., (Machines Without Principals: Liability Rules and Artificial Intelligence, Washington Law Review 20I4, II7) realiza un profundo análisis de las regas aplicables a los "accidentes inexplicables", respecto de los cuales resulta aplicable el canon res ipsa loquitur: este último, de hecho, deduce la existencia de un defecto y de que existan numerosos y frecuentes casos en los que se haya manifestado un funcionamiento defectuoso, también cuando la causa tecnológica del hecho no haya sido establecida. En nuestra hipótesis, por el contrario, los accidentes resultan raros, además de no atribuibles a un defecto preciso. 
cierta tecnología llegue al mercado, porque una regla similar generaría un desincentivo a la completa automatización; adicionalmente, tampoco estaría en línea con la ratio que fundamenta la responsabilidad del fabricante, que radica en el hecho de estar en una posición más ventajosa para soportar los costos de las acciones resarcitorias. La incertidumbre, desde el punto de vista tecnológico además del jurídico, hasta tanto no se absuelva el problema relativo a la aplicación de las reglas existentes a estas nuevas realidades-, es tal que no permite una estimación, de manera que el riesgo resulta difícilmente asegurable, o lo es a un costo desproporcionado respecto a la creciente seguridad de los sistemas.

En un escenario de auténtico replacement ${ }^{89}$, donde los robots sustituirán al hombre en algunas actividades, dado que ellos aumentan la eficacia y la seguridad de funcionamiento, es plausible que se deban introducir esquemas alternativos. Además de aquellos ya examinados, han sido identificados otros modelos que van desde la construcción de una regla de responsabilidad objetiva ${ }^{\circ}$, a los sistemas sin culpa, diversamente sustentados, a la responsabilidad de todos los sujetos económicos involucrados en la puesta en funcionamiento de los diversos componentes de un sistema o, incluso, al simple régimen de seguros individuales, puesto que los accidentes serían tan extraños que harían que la prima del seguro fuera muy baja y, por lo tanto, accesible a todos los consumidores privados. La creación de una personalidad jurídica de la máquina, asociada a un patrimonio que podría estar alimentado por diversas fuentes de financiamiento, constituye, en definitiva, una hipótesis que es constantemente explorada por los estudios en la materia ${ }^{91}$.

La definición de los instrumentos jurídicos que podrían acompañar los desarrollos tecnológicos, en el camino hacia una completa automatización, es una obra en curso, limitada, pero cierta en algunos aspectos. Entre estos, que el estudio de una solución eficiente para el problema clásico del costo de los accidentes no debe separarse de las valoraciones sobre la oportunidad de la sustitución del hombre por el robot para el desarrollo de funciones hasta el momento típicamente humanas. En segundo lugar, que la eventual elección de reglas que incentiven algunas tecnologías robóticas no puede prescindir de su mayor demanda social, por su correspondencia con finalidades calificadas o, incluso, con el cumplimiento de objetivos impuestos al legislador ${ }^{92}$.

89 Kerr, I., en Robot Law, cit. Sobre el tema del replacement en la actividad de cuidado mediante agentes artificiales ver, por el contrario, Coeckelbergh, M., Artificial agents, good care, and modernity, en Theoretical Medicine and Bioethics, 2015 (36), 265-277.

90 Es la propuesta de VLADECK, últ. ob. cit., I 46 ss., que a ella asocia una suerte de "court-compelled insurance" para responder a las finalidades de compensación y a los costos transaccionales.

9I El Draft Report del IURI Committee del Parlamento europeo (punto 3 I) enumera los posibles enfoques y configura la subjetividad de los robots autónomos, como el punto extremo de una progresión ideal hacia reglas innovativas de responsabilidad.

92 Desarrolla este razonamiento con relación a las prótesis robóticas, p. ej., A. BerTolini, "Robotic prostheses as products enhancing the rights of people with disabilities. Reconsidering the structure of liability rules", International Review of Law Computers and Technology, 20 I 5, 29 (2-3), I I6- I 36. 


\section{Cyborgs y human enhancement entre viejo...}

El tema de la fusión hombre-máquina y de la potencialización humana93 es una conclusión natural de la búsqueda de la interacción entre robótica y derecho. La robótica es, en efecto, uno de los medios tecnológicos más potentes para lograr el crecimiento de las capacidades humanas, que se lleva a cabo por medio del implante de artefactos en el cuerpo o bien, desde las perspectivas más radicales y futuristas, mediante el abandono del cuerpo y la continuación de la personalidad en un soporte artificial. En la situación actual, la puesta en funcionamiento de prótesis avanzadas y de exoesqueletos robóticos está dirigida a auxiliar en forma eficiente a personas afectadas por una discapacidad motora o a volver más fáciles y seguros los movimientos de los ancianos. De la misma forma, implantes cocleares, ampliamente difundidos en el uso clínico, o implantes de retina, todavía en fase de experimentación, permiten restituir capacidades auditivas o visivas a las personas que se encuentran impedidas a causa de traumas o malformaciones. Es más, implantes para la estimulación cerebral ya se utilizan para el tratamiento de síntomas como el temblor en el mal de Parkinson, y se está probando su implementación para el tratamiento de otras condiciones patológicas como el Alzheimer, la depresión y la dependencia de sustancias estupefacientes.

Interfaces cerebro-máquina pueden ser utilizadas tanto para que personas que están imposibilitadas para comunicarse con el exterior puedan hacerlo, como en el caso de la locked-in síndrome, como para controlar dispositivos como prótesis, sillas de ruedas y otras plataformas robóticas. Otras tipologías de implantes se encuentran en fase de estudio a fin de superar déficits cognitivos y consentir la recuperación de determinadas funciones, como por ejemplo aquellas ligadas a la adquisición y a la conservación de los recuerdos. Estos objetivos de carácter esencialmente terapéutico ya dejan entrever posibles evoluciones en cuanto a la potencialización en el plano físico, sensorial y cognitivo. Prótesis u otros sistemas robóticos, directamente interconectados con el sistema nervioso, podrán desarrollar en manera eficiente las mismas funciones que las extremidades naturales, pero también potencializar las actuales capacidades humanas, por ejemplo, permitiendo cumplir con acciones a distancia y superar, por ende, vínculos espaciales; o bien, aumentar la fuerza e intensidad de los propios gestos; o, incluso, actuar en la escala temporal habitual, puesto que el pensamiento puede accionar un dispositivo mecánico en modo acelerado respecto al normal proceso neuronal que mueve un brazo o una pierna94. También la potencializa-

93 Recientemente, sobre el tema de la potencialización humana, a modo de síntesis del debate actual, ver Palazzani, L., Il potenziamento umano. Tecnoscienza, etica e diritto, Torino, 2015.

94 Nicolelis, M., Il cervello universale. La nuova frontiera delle connessioni tra uomini e computer (trad. ital. de A. Panini de Beyond Boundaries: The New Neuroscience of Connecting Brains with Machines and How It Will Change Our Lives, New York, 201 I), Torino, 2013, I 84 ss. 
ción cognitiva, normalmente obtenida mediante la asunción de fármacos u otras sustancias, puede realizarse por medio de implantes neuronales u otros sistemas no implantables que generan campos magnéticos capaces de estimular la actividad cerebral.

Esta variedad de sistemas serán dotados de sensores para percibir al ambiente real y estarán conectados a la red de internet, en modo de permitir a quien los lleva consigo recoger y procesar información con una magnitud que de lo contrario no podría ser percibida por la experiencia humana y, de este modo, por ejemplo, resolver cálculos matemáticos complejos, sobresalir en habilidades lingüísticas o generar destrezas musicales. Algunos estudiosos predicen que la convergencia de la robótica y de las neurociencias llevará a la puesta en funcionamiento de neuroprótesis avanzadas, que permitan a los tecno-científicos usar la tecnología digital, informativa y neuroscientífica como un auxilio permanente 95 ; y que en el curso de pocos años, el desempeño de sistemas híbridos hombre-robot superará el de los sistemas puramente biológicos ${ }^{6}$. Se trataría del primer paso hacia una transformación transhumanista, que verá a los hombres convivir con híbridos hombre-máquina, hasta poder ser sustituidos por robots $\mathrm{u}$ otra suerte de sistemas mecánicos a los cuales transferir, con un acto de upload, la conciencia humana y lograr de esta manera una especie de inmortalidad 97 .

Estos escenarios requieren un doble nivel de análisis: por un lado, a fin de identificar el régimen aplicable al cuerpo objeto de la transformación y al aparato tecnológico que, integrado con el cuerpo, termina por desarrollar funciones que a este le son útiles o esenciales; por otro lado, a los fines de definir los propios límites -si es que los hay- de las posibilidades de transformación o deformación del cuerpo, al punto de superar la prospectiva de los confines del plano físico.

En relación con ambos interrogantes, la distinción, aunque fuertemente controvertida, entre alteraciones terapéuticas y acciones dirigidas a la potencialización humana, está destinada a desempeñar un rol esencial. De hecho, por un lado, no se puede dudar de que la modificación y la inclusión de artefactos en el cuerpo sean legítimas, cuando tales procedimientos presentan naturaleza intrínsecamente terapéutica. Los ejemplos de este tipo de alteraciones son diversos y conocidos en forma previa a los desarrollos más prometedores de la robótica: desde el uso de órganos y tejidos artificiales para el sostenimiento de funciones vitales a través de auxilios mecánicos, bien sean externos al cuerpo, como en la

95 Wallach, From Robots, cit., I 86.

96 Nourbaknsh, I. R., The Coming Robot Dystopia, en Foreign Affairs, I 6 de junio de 20 I 5, disponible en: https://www.foreignaffairs.com/articles/201 5-06-r6/coming-robot-dystopia

97 KurzweIL, R., Singularity is Near: When Human Transcends Biology, New York, 2005; Chalmers, D. J., The Singularity: A philosophical analysis, en fournal of Consciousness Studies 2010, I 7(9-10), 7-65. Recientemente, un estudio detallado del argumento, incluso de las opiniones críticas, ha sido proporcionado por los análisis reunidos en el volumen Intelligence Unbound: The Future of Uploaded and Machine Minds, Blackford, R. y Broderick, D. (eds.), Chichester, 20 i4. 
ventilación mecánica, o implantados, como el pacemaker o las bombas para la insulina. De igual manera, las hipótesis en las que la intervención terapéutica es precedida por una operación extirpadora, como es el caso de la amputación selectiva de una extremidad (lesionada por un trauma) seguida de una reconstrucción biónica ${ }^{9}$, no exceden la esfera precedida por el interés de la tutelada de la salud de las personas.

Por otro lado, el cuerpo transformado podría continuar sujeto al mismo régimen del cuerpo puramente biológico, cuando la transformación se produce en consonancia, e incluso a favor de un objetivo ligado a la tutela de la salud o de otro interés fundamental. A esta conclusión se dirigen tanto el análisis conceptual como la praxis jurisdiccional que ha sabido afrontar las consecuencias de la desaparición de los límites entre cosa y persona generados por el desarrollo tecnológico, en forma coherente con la garantía de los derechos fundamentales. Con su implante en el cuerpo y su destinación a funciones esenciales, el dispositivo artificial es absorbido por el régimen de las partes naturales correspondientes, puesto que esto depende del rol instrumental que este desempeña en relación con la preservación de la vida, con la promoción de la salud y con la protección de la identidad de la persona. En forma muy subjetiva, y no necesariamente en relación con la ambigüedad de estatus introducida por el progreso tecnológico, se ha afirmado que "la mente no coincide con el espacio corporal, sino que lo incluye dentro de sí; y entonces, espacio interno y espacio externo pueden realizar un proceso de osmosis en la construcción del yo. El yo puede impregnar fuertemente algunos objetos extraños que por memoria, afectos, necesidad, son vehículos insustituibles y canales de experiencia e incluso de percepción de sí mismo"99.

Además de las hipótesis de “cuerpos extendidos”, en los cuales la calificación y el régimen propio de la corporeidad se aplican a cosas que están materialmente "fuera" de ella ${ }^{100}$, en la práctica también se conocen otras hipótesis de extensiones o sustituciones robóticas que han sido resueltas sin producir efectos disruptivos en las categorías conceptuales involucradas. En una controversia surgida entre un veterano estadounidense cuadripléjico y la compañía aérea cuya tripulación dañó su silla de ruedas robótica, la naturaleza del dispositivo determinó la decisión de la empresa aseguradora de conceder un resarcimiento calculado

98 Aszmann, O., et al., "Bionic reconstruction to restore hand function after brachial plexus injury: A case series of three patients", Lancet online, 25 de febrero de 20 I 5 .

99 ZAтTі, P., "Di là dal velo della persona fisica. Realtà del corpo e diritti dell'uomo", en Maschere del diritto, volti della vita, Milano, 2009, 63.

Iо० La referencia es al conocido caso decidido por BGHz I 24, 52 vi. Civil Senate (vi zR 62/93), 9 de noviembre de I993, donde la destrucción de la muestra de esperma congelada, por parte de la clínica donde estaba conservada, dio lugar al resarcimiento de los daños a la integridad física en los términos del $\$ 823$ г в вв, en la medida en que la muestra de esperma es considerada parte del cuerpo donante. 
en el valor de las lesiones físicas y no del daño de un bien material. De hecho, el mobility assistance device (MAD) usado por la víctima era un aparato mucho más sofisticado que una silla de ruedas común, constituyendo una suerte de prótesis que sustituye las extremidades inferiores y los músculos del torso, de manera que durante el largo período que fue necesario para su reparación, la persona discapacitada estuvo obligada a permanecer en cama ${ }^{\mathrm{IOI}}$. De ahí que la máquina no pudiera ser tratada como un mero objeto de propiedad de la víctima, si de esta calificación se derivan límites al resarcimiento de los daños sufridos por la víctima.

Una predisposición genética como el daltonismo, que solo permite ver en blanco y negro, se corrige mediante un "eyeborg", esto es, un dispositivo constituido por una telecámara permanente ubicada en la cabeza, que convierte a los colores en ondas sonoras en tiempo real, permitiendo percibir, mediante diversas frecuencias, la gama de tonalidades existentes. La posibilidad de llevar consigo este dispositivo en la fotografía que aparece en el documento de identidad está en el centro del debate de las autoridades británicas ${ }^{102}$, circunstancia que evidencia la novedad de las problemáticas, también de tipo administrativo, planteadas por el progreso tecnológico, pero al mismo tiempo la apertura progresiva de las nociones de cuerpo y de identidad personal.

Por lo tanto, en el estado actual de la legislación y de la interpretación jurisprudencial existe un respaldo jurídico para la inclusión de la tecnología en el cuerpo, cuando su uso satisface intereses existenciales. Las opiniones escépticas sobre la existencia de medidas de protección suficientes, que se puedan extender de la persona a los aparatos de prótesis que asisten funciones biológicas ${ }^{\mathrm{IO} 3}$, no consideran las potencialidades evolutivas de un sistema de protección que no se basa de manera estricta en la materialidad corpórea, sino en la dimensión de los intereses que recaen sobre la corporeidad.

Sin embargo, existen otros tipos de riesgos frente a los cuales la disciplina actual no siempre está en condiciones de ofrecer una protección adecuada. El principal está representado por las potenciales interferencias externas sobre los dispositivos instalados en el cuerpo, los cuales, cada vez más con mayor frecuencia, se encuentran dotados de una conexión a la red que, por lo tanto, los hace

Iо I Un animado informe proporcionado por el abogado que defendió al veterano contra la compañía aérea en McDonald Glenn, L., Case Study: Ethical and Legal Issues in Human-Machine Mergers (Or the Cyborgs Cometh), en Annals of Health Law ALSME Special Edition, 201 2, 2 I, I 75I80 (http://ssrn.com/abstract=2 102425).

IO2 Para un informe autobiográfico de la experiencia, ver: http://www.ted.com/talks/neil_ harbisson_i_listen_to_color

I03 Expresada, p. ej., por Wittes, B. y Chong, J., Our Cyborg Future. Law and Policy Implications, Center for Technology Innovation at Brookings, setiembre de 201 5, disponible en: http://www. brookings.edu/research/reports2/2014/og/cyborg-future-law-policy-implications 
vulnerables a los cyber-ataques ${ }^{104}$. De igual manera, como ya se expuso, ni la normatividad actual en materia de dispositivos médicos (incluso el proyecto de revisión de la normatividad existente) ${ }^{\mathrm{IO} 5}$ ni la disciplina penal ${ }^{\mathrm{IO} 6}$ parecen estar en posición de enfrentar formas de agresión, que sin implicar un contacto físico se encuentran en capacidad de lesionar la integridad física y la salud, así como la intimidad de las personas que llevan consigo implantes interconectados con la red. Las reglas generales de responsabilidad civil y de responsabilidad por productos defectuosos podrían ser aplicadas cuando se presente un mal funcionamiento del dispositivo, causado por una agresión virtual; sin embargo, ello no garantiza la seguridad que el consumidor podría esperar. Estas últimas implican la comparación entre la fiabilidad del aparato y un conjunto ideal de medidas que habrían podido evitar el daño, probablemente, incidiendo al mismo tiempo en la provisión de sistemas de protección en la fase de producción y puesta en funcionamiento de estos dispositivos. Ciertamente, los requisitos de cyber-seguridad deberían completar el conjunto de reglas técnicas preventivas, que preceden a la circulación de instrumentos activos de ayuda tecnológica para controlar su seguridad y eficiencia.

Finalmente, otra doctrina advierte ulteriores ocasiones de daño en el uso de tecnologías de asistencia a la movilidad, la percepción o la comunicación, que derivan del ejercicio de derechos de propiedad sobre los productos en cuestión o sobre sus fundamentos técnicos. Se plantea, en específico, que mediante acuerdos de licencia detalladamente elaborados se pueden limitar los usos de una prótesis o de otro aparato, de forma tal que quien los utilice encuentre restricciones en lo que puede hacer con elementos que se convirtieron en parte de su cuerpo $^{107}$. De esta manera se vería indirectamente afectada la libertad de acción de la persona, aunque esta no esté físicamente sujeta a restricciones. Esta perspectiva señala, oportunamente, los problemas inéditos que genera el implante de productos tecnológicos en el cuerpo, que al mismo tiempo están protegidos mediante derechos de propiedad intelectual. Esta tesis simplifica, de manera bastante persuasiva, la conclusión del desvanecimiento de los confines entre cosa y persona, y con ella se fundamenta, una vez más, la necesidad de extender el estatus de la persona a los objetos, en función de su pertenencia a la vida material de la persona y a su instrumentalidad respecto a sus intereses vitales. En el plano técnico, esta tendencia estararía respaldada, además, por el régimen general

I04 Wittes y Chong, ob. cit.; Wu, S. S. y M. Goodman, "Neural Devices Will Change Humankind: What Legal Issues Will Follow?”, The SciTech Lawyer, 20 1 2, 8(3).

I05 Ver supra notas 4I-45 y el texto correspondiente.

Io6 Koops, B.-J. y Gasson, M. N., "Attacking Human Implants: A New Generation of Cybercrime", en Law, Innovation and Technology, 2013, 5(2), 248-277.

io7 Ramachandran, G., "Against the Right to Bodily Integrity: Of Cyborgs and Human Rights", 87 Denver University Law Review 2009, I-57, 9 ss. 
de los contratos, que, al sancionar el contenido de la reglamentación privada que contrasta con la ley o con los principios de orden público, puede lograr la remoción de las cláusulas que tienen por efecto limitar la libertad o un derecho fundamental de la persona.

En consecuencia, el sistema jurídico actual parece estar en capacidad de asegurar cobertura a las exigencias de protección que nacen de la compenetración entre el hombre y los artefactos tecnológicos, al menos cuando esta se dirige a garantizar, de la forma más eficaz, intereses fundamentales. Por otra parte, el ordenamiento también debe proteger, de manera reflexiva, el rechazo de la tecnología, aun cuando esté orientada a la superación de discapacidades, y ofrecer alternativas a las soluciones puramente tecnológicas ${ }^{108}$.

\section{VIII. ... y nuevo}

Solo recientemente el término cyborg comienza a ser cuestionado en la reflexión ética y jurídica. Una versión débil de esta tendencia únicamente alude al uso de la tecnología como auxilio de la superación de patologías y discapacidades, mediante el implante de un dispositivo externo en el cuerpo humano. Desde el implante coclear, a la estimulación cerebral profunda y a la prótesis avanzada, la combinación de instrumentos electrónicos y meca-trónicos con materialidad corpórea no da lugar a cuestiones que resulte imposible resolver desde el punto de vista jurídico. Respecto a los auxilios tradicionales, como un pacemaker, las problemáticas aumentan en el plano cuantitativo, asociadas a la mayor complejidad del sistema nervioso con la que estos dispositivos están interconectados, con la delicadeza de la intervención requerida para su posicionamiento $y$, a veces, con la necesidad de una fase de entrenamiento para aprender su uso. Ciertamente, el cyborg, entendido como el hombre que mediante el uso de un aparato tecnológico supera déficits físicos, perceptivos y motores, no constituye una novedad radical para el sistema jurídico.

A pesar de la naturaleza prevalentemente terapéutica de estos dispositivos, en los mismos se entrevé un potencial ulterior: la telecámara para "sentir" los colores permite captar también los rayos infrarrojos, no accesibles al ojo humano; el brazo robótico no solo permite ejercitar la función prensil, sino que le imprime mayor fuerza a la acción o permite superar otros vínculos espaciales y temporales; el implante visual, por el momento solo en fase experimental, o el auditivo, podrían conferir capacidades perceptivas definitivamente superiores a las comunes. La tecnología robótica representa un instrumento eficaz para la

ro8 Piénsese en los implantes cocleares para la curación de ciertas formas de sordera, que a diferencia de otros tratamientos se insertan quirúrgicamente dentro del oído medio. Una parte importante de sus potenciales destinatarios los rechaza porque no conciben la sordera como una incapacidad, sino como una diferencia que es parte integrante de la identidad y, como tal, debe ser protegida. 
enhancement a todo nivel, físico, sensorial y cognitivo, de modo tal que se acerca a la prospectiva de la potencialización humana más allá de cualquier finalidad, siquiera parcialmente terapéutica. El cyborg, en una segunda acepción del término, es el hombre que gracias a un equipamiento tecnológico supera los límites físicos y mentales propios de la psiquis y de la propia corporalidad humana; pero también aquel que confía a la máquina las tareas automáticas que una computadora puede desarrollar de manera rápida y eficiente, para concentrarse en funciones superiores, creativas e intelectualmente exigentes. En la versión más radical de esta expresión y de la ideología a la que está ligada, el cyborg es la entidad que se libera del cuerpo, con todas sus debilidades, para continuar viviendo en un soporte únicamente artificial, virtual o mecánico.

Los interrogantes suscitados por estos escenarios futuristas son múltiples, y se mueven, principalmente, entre la identificación del estatus aplicable a sistemas híbridos hombre-máquina y la misma legitimación de la transformación de la condición humana para ser incorporada en seres virtuales, pasando por la aceptabilidad de un uso ilimitado de instrumentos artificiales para atravesar las fronteras del plano físico. ¿Puede admitir el derecho una corporeidad cambiante, fluida, ni natural ni artificial, ni orgánica ni mecánica? ¿̇ existen en el sistema constitucional límites que se refieren a un núcleo constitutivo, identificativo, estructural y, por lo tanto, irrenunciable de la identidad humana en el cuerpo?

Las respuestas a estos interrogantes deben ser necesariamente normativas y radicarse en un sistema de valores. De hecho, los escenarios descritos, aunque totalmente futuristas, no parecen encontrar límites en la biología, por lo menos si se tiene en cuenta la hipótesis del enhancement humano, a través de un equipamiento anexo al cuerpo, que pasa a formar parte del mismo en forma permanente. Los estudios que han sido conducidos hasta las fronteras más avanzadas de la neurociencia, prácticamente califican como necesarias estas posibles transformaciones: con la adquisición de la capacidad de usar instrumentos artificiales, el cerebro comienza a considerarlos como verdaderas extensiones del cuerpo biológico y a crear una representación en los circuitos neuronales. Mediante un proceso altamente dinámico y de adaptación, el sistema nervioso central puede contribuir a diseñar una imagen del cuerpo modelada con base en las informaciones visivas, táctiles y sensoriales que recibe, hasta el punto de producir un sentido de pertenencia corpórea que puede "inducir a cada uno de nosotros, en pocos segundos, a aceptar otro cuerpo enteramente nuevo como si fuera la verdadera casa de nuestra existencia consciente" ${ }^{\circ 09}$. La capacidad expansiva y de mejoramiento de la tecnología robótica puede, por lo tanto, ser asimilada en nuestra corporeidad de modo prácticamente natural, extendiendo materialmente nuestras posibilidades de acción y de interacción con el ambiente.

Io9 Nicolelis, ob. cit., 84 s. 
Por el contrario, en el plano normativo, una constatación formulada de tiempo atrás -con la habitual apertura visual hacia el futuro- y según la cual "los 'derechos humanos' no estarían perdidos en una transición eventual hacia el post-humano o el trans-humano [...] El derecho se asoma a estos nuevos territorios y de ellos no puede sustraer la mirada" Iо , constituye un punto de partida, pero la aspiración de delinear una condición poshumana en su relación con los derechos fundamentales está todavía por construirse.

Una primera prospectiva confiaría exclusivamente a la autonomía individual la elección de recurrir a la tecnología para fines de enhancement. Es más, la transformación del cuerpo mediante la técnica está siendo cada vez más conceptualizada como objeto de un derecho, una posibilidad abierta que se vuelve parte del derecho de construir libremente la propia identidad, usando todas las oportunidades a disposición ${ }^{\mathrm{II} \text { I-II2}}$.

Sin embargo, la regulación no puede cincunscribir el problema del mejoramiento humano a la esfera privada, puesto que los efectos de las elecciones personales recaerían, en combinación con la difusión de experiencias similares, sobre la comunidad. Ello podría suceder incluso de manera directa, cuando una capacidad especial con la que decide dotarse una persona -piénsese en la visión de rayos $\mathrm{X}^{\mathrm{II}}$ - pueda ser usada para violar derechos de otros (en este caso el derecho a la intimidad). Pero también en modo más sutil e indirecto, dado que un fenómeno de amplia dimensión podría afectar el orden social, provocando la distorsión de valores sobre los que este se basa, como la igualdad y la no discriminación, la justicia distributiva, la igualdad de oportunidades ${ }^{\mathrm{II}}$; o bien terminaría por restringir la libertad de aquellos que preferirían prescindir de las

I IO Rodotà, S., La vita e le regole. Tra diritto e non diritto, Milán, 2006, 87.

I I I Rodotà, S., "Of machines and men: The road to identity. Scenes for a discussion”, en HiLdebrandt, M. y Rouvroy, A. (eds.), Law, Human Agency and Autonomic Computing. The Philosophy of Law Meets the Philosophy of Technology, Oxford-New York, 20 I I, I80.

I 2 Un centro de investigación californiano, el Institute for the Future, elaboró un documento destinado a ofrecer un cuadro normativo para el enhancement cognitivo, titulado Magna Cortica, que incluye un derecho a la auto-transformación para la potencialización cognitiva, así como un derecho de rechazarla, que debe estar garantizado aunque tal elección podría con el tiempo ser considerada irresponsable. Ver: http://www.openthefuture.com/20 I4/o5/magna_cortica.html

I 3 El ejemplo es de Brownsword, R., "Regulating Human Enhancement: Things Can Only Get Better?", en Law, Innovation and Technology, 2009, I, I42.

I 4 No por casualidad, partiendo de una perspectiva individualista se resalta en todo caso la necesidad de reformular el contenido de los derechos fundamentales, o bien crear otros nuevos, ya sea para los individuos potencializados, ya sea para todos los demás: Koops, B.-J., "Concerning 'Humans' and 'Human' Rights. Human Enhancement from the Perspective of Fundamental Rights", en Engineering the Human. Human Enhancement Between Fiction and Fascination, Koops, B.-J.; Lüthy, C.H.; Nelis, A.; Sieburgh, C.; Jansen, J. P. M. y Schmid, M. S. (eds.), BerlinHeidelberg, 2013, I65-182. 
oportunidades ofrecidas por el progreso tecnológico, pero que sufren el condicionamiento derivado del uso masivo que hacen los demás ${ }^{\mathrm{II}}$.

Al mismo tiempo, una matriz estrictamente utilitarista, abierta a toda innovación que contribuya a maximizar el progreso en búsqueda de un mayor bienestar colectivo y que, en consecuencia, aprueba por definición el buman enbancement que se propone optimizar lo humano ${ }^{116}$, no resulta dirimente. Por una parte, la fragilidad y la dependencia que se pretende superar, así como las relaciones de solidaridad y de cuidado que aquellas generan, detentan un valor en nuestra sociedad ${ }^{117}$; y la diversidad, la imperfección, la pluralidad de las cualidades humanas enriquecen la experiencia y son consideradas elementos que se deben preservar ${ }^{\mathrm{II} 8}$. Por otra parte, el objetivo último de la invulnerabilidad no es alcanzable de manera realista. De algún modo, si la tecnología puede ayudar a disminuir el sufrimiento, siempre creará nuevas formas de vulnerabilidad, como por ejemplo la mayor dependencia respecto de los sistemas biológicos y tecnológicos que se implantan y expanden la mente ${ }^{\mathrm{II} 9}$. Además, renunciando a la dependencia hacia los demás, gracias a la tecnología, se renuncia también a la dimensión de la socialización, propia de los seres humanos.

De esto se deriva que el fenómeno debe ser considerado a la luz de los valores en los cuales se basa la sociedad europea, orientando el discurso a las nuevas desigualdades, entre individuos naturales e individuos "aumentados", que son fruto de la potencialización tecnológica; de la tensión entre la búsqueda de la justicia social en el acceso a los recursos tecnológicos y el respeto de la dignidad de la persona. El horizonte argumentativo que se abre con esta última noción es muy amplio, y la multiplicidad de sus significados -que prácticamente llegan a incluir a sus opuestos- es una de las características que la distinguen. Por lo tanto, debe hacerse un uso muy moderado y atento de los mismos, especialmente para decidir sobre cuestiones que todavía no han mostrado su verdadera potencialidad, en sentido benéfico o dañoso. Adicionalmente, las cartas de derechos fundamentales más recientes han sido diseñadas, precisamente, para identificar y combatir prácticas contrarias a la dignidad humana, mediante la cooperación entre Estados y organismos supranacionales ${ }^{\mathrm{I2O}}$; finalmente, en el conjunto de

I 5 Para algunos ejemplos bastante plausibles, como la incidencia de las técnicas anti-envejecimiento sobre la edad para jubilarse, ver además Koops, “Concerning 'Humans' and 'Human' Rights", cit., I 75 ss.

i 6 Harris, J., Enhancing evolution: The ethical case for making better people, Princeton, 2007.

I 7 Nussbaum, M., Giustizia sociale e dignità umana. Da individui a persone, Bolonia, 2002.

i 8 Fukuyama, F., Transhumanism, en Foreign Policy, 23 de octubre de 2009.

i 9 Coeckelbergh, M., Human Being @ Risk. Enhancement, Technology, and the Evaluation of Vulnerability Transformations, Dordrecht, 2013 ; Id., Vulnerable Cyborgs: Learning to Live with our Dragons, en Fournal of Evolution and Technology, 201 I , 22, I-9.

I $20 \mathrm{El}$ artículo I I de la Unesco Declaration on the Human Genome and Human Rights, del i I de noviembre de I997, prevé: "Practices which are contrary to buman dignity, such as reproductive cloning 
valores que caracteriza a la Carta de Niza, las referencias a la dignidad sobresalen en los mecanismos de tutela concebidos frente a las eventuales agresiones a la persona y a la corporeidad en el campo de la biología y de la medicina. Como también lo demuestra la prohibición de clonación reproductiva, de dimensión universal y presente en los dos planos citados, uno de los posibles significados de la palabra insiste en la irreductible unidad de cada individuo; en el cuerpo como factor de identificación y de diferenciación frente a los demás. En este escenario, no parecen compatibles con la dignidad de la persona las intervenciones que tengan como objetivo la reducción de la diversidad humana y biológica, la superación de las características excéntricas que se alejen de la normalidad, o la uniformidad de la especie, aunque las mismas estén orientadas a la potencialización de su estructura biológica.

En este sentido, algunos fragmentos del sistema italiano también parecen estar predispuestos para oponer una resistencia a la transformación del cuerpo dirigida a trascender la biología y la propia cualidad de ser limitado, como lo es el hombre. Esta idea subyace a la prohibición del trasplante de las gónadas y del encéfalo, contenida en la ley italiana relativa al trasplante de órgano de cadáveres (art. 3, inc. 3 Ley n. ${ }^{\circ}$ 9I del r. ${ }^{\circ}$ de abril de r999), la cual expresa la idea, de manera prácticamente ingenua, de que estas partes del cuerpo están ligadas, más que otras, a la identidad de la persona, de modo tal que no son susceptibles de trasplantes, como por el contrario lo son otros órganos, $y$, en consecuencia, no debería renunciarse a ellos ni siquiera con miras a la creación de hombresmáquina dotados de una inteligencia artificial; o, según otra propuesta, de una especie nueva, despojada de la forma humana, incluida la diferencia de género ${ }^{\mathrm{I} 2 \mathrm{I}}$. Se trata de un ejemplo aislado, de reglas tal vez "platónicas" o "vanas" -en el sentido de que no encontrarán fácilmente ocasión de ser aplicadas- pero que no dejan de revestir la condición de normas-símbolo ${ }^{122}$, capaces de ordenar, en el marco de la dignidad, el inicio de un discurso poshumano.

Tampoco faltan las afinidades con la concepción habermasiana, que vincula a la dignidad y a la especificidad de la identidad humana el rechazo de técnicas como la manipulación genética, que privarían al individuo de su derecho a un futuro abierto y no condicionado, convirtiéndolo de esta forma -no importa en qué medida, aunque sea mínima- en objeto de disposición y determinación por parte de los demás ${ }^{\mathrm{I2} 3}$. En este sentido, seguramente, la dignidad se opone a las versiones más extremas de la visión transhumanista, que imagina la creación de

of human beings, shall not be permitted. States and competent international organizations are invited to co-operate in identifying such practices and in talking, at national or international level, the measures necessary to ensure that the principles set out in this Declaration are respected".

I 2 Dagnino, A., Uoma. La fine dei sessi, Milán, 2000.

I 2 ZATti, P., Note sulla semantica della dignità, en Maschere del diritto, cit., 30.

I 23 Habermas, J., The Future of Human Nature, Cambridge, 2003. 
seres artificiales que no pueden ser libres, en la medida en que son desprovistos de la libertad y de la casualidad en el momento genético.

Si los principios constitucionales diseñan un marco que sirve para argumentar la discusión teórica en el momento práctico, la dicotomía, controvertida y cargada de problemáticas, entre terapia y potencialización ${ }^{124}$ sigue siendo irrenunciable. Ciertamente, aquellos no pueden servir para discriminar, de un modo simplista, lo que es lícito con respecto a lo que no lo es, solo por la incertidumbre definitoria que encierran las dos nociones; la fragilidad de las fronteras que las separan y la aceptación, prácticamente pacífica, de prácticas invasivas -como la cirugía estética-, que se ubican en el campo del mejoramiento y no de la terapia. Sin embargo, si no es posible decidir sobre la licitud de las intervenciones realizadas en el cuerpo, no se puede descartar completamente la admisibilidad de un discurso fundado en la finalidad terapéutica del acto. Cuando el objetivo de dotarse de un aparato tecnológico es el de recuperar funcionalidades perdidas, se está frente a una base incontrovertible como el derecho a la salud; a falta de este, se debe discutir, al menos, si la misma aspiración debe ser satisfecha empleando recursos como la profesionalidad médica, y si la misma deontología profesional y el marco proporcionado por la finalidad de la medicina puedan señalar los límites ${ }^{\mathrm{I} 25}$.

Mientras la discusión ética y jurídica continúa, comprometiendo además el plano de las políticas públicas ${ }^{\mathrm{I} 26}$, es posible que la praxis asigne a los comités de ética de las entidades hospitalarias la tarea de una valoración caso por caso, lo que tendría el mérito de señalar el estado del arte en la materia y determinar el avance del derecho sin traumas y sin barreras demasiado autoritarias.

I 24 Ver la discusión al respecto en los estudios reunidos por Lucivero y Vedder, Beyond Therapy $v$. Enhancement?, cit.

I25 Schermer, M., Health, Happiness and Human Enhancement. Dealing with Unexpected Effects of Deep Brain Stimulation, en Neuroethics, 2013 3, 6, 435-445.

in 6 ter Meulen, R., Human Enhancement: A Policy Perspective for the European Union, en Boer, T. y Fischer, R. (eds.), Human Enhancement. Scientific, Ethical and Theological Aspects from a European Perspective, Strasbourg, 201 3; Coenen, S.; Schuijff, M. y Smits, M., "The Politics of Human Enhancement and the European Union”, en Savulescu, J.; ter Meulen, R. y Kahane, G. (eds.), Enhancing Human Capacities, Oxford, 20 I I, 52 I-535; President's Council on Bioethics, Beyond Therapy. Biotechnology and the Pursuit of Happiness, 2003. 